\title{
Longitudinal Vibration of Isotropic Solid Rods: From Classical to Modern Theories
}

\author{
Michael Shatalovi,2, Julian Marais², \\ Igor Fedotov ${ }^{2}$ and Michel Djouosseu Tenkam² \\ ${ }^{1}$ Council for Scientific and Industrial Research \\ ${ }^{2}$ Tshwane University of Technology \\ South Africa
}

\section{Introduction}

Longitudinal waves are broadly used for the purposes of non-destructive evaluation of materials and for generation and sensing of acoustic vibration of surrounding medium by means of transducers. Many mathematical models describing longitudinal wave propagation in solids have been derived in order to analyse the effects of different materials and geometries on vibration characteristics without the need for costly experimental studies. The propagation of elastic waves in solids has seen particular interest since the end of the 19th century. The solution for three dimensional wave propagation in solids was derived independently by L. Pochhammer in 1876 and by C. Chree in 1889. The solution describes torsional, longitudinal and flexural wave propagation in cylindrical rods of infinite length and is known as the Pochhammer-Chree solution (Achenbach, 1999:242-246; Graff, 1991:470473). The Pochhammer-Chree solution is valid for an infinite bar with simple cylindrical geometry only. For even slightly more complex geometry, such as conical, exponential or catenoidal, no exact analytical solution exists. The need for useful analytical results for bars with more complex geometries fuelled the development of one dimensional approximate theories during the 20th century. The exact Pochhammer-Chree solution has typically been used as a reference result in order to make deductions regarding the accuracy of the approximate theories and the limits of their application (Fedotov et al., 2009).

The classical approximate theory of longitudinal vibration of rods was developed during the 18th century by J. D'Alembert, D. Bernoulli, L. Euler and J. Lagrange. This theory is based on the analysis of the one dimensional wave equation and is applicable for long and relatively thin rods vibrating at low frequencies. Lateral effects and corresponding lateral and axial shear modes are fully neglected in the frames of this theory. The classical theory gained universal acceptance due to its simplicity, especially for engineering applications. It is broadly used for design of low frequency mechanical waveguides such as ultrasonic transducers, mechanical filters, multi-stepped vibrating structures, etc.

J. Rayleigh was the first who recognised the importance of the lateral effects and analysed the influence of the lateral inertia on longitudinal vibration of rods. This result was briefly exposed in Rayleigh's famous book "The Theory of Sound", first published in 1877 (Rayleigh, 1945:251-252). A. Love in his "Treatise on the Mathematical Theory of Elasticity", first published in 1892 (Love, 2009:408-409), further developed this theory, which is now 
referred to as the Rayleigh-Love theory. The lateral inertia effects are important in the case of non-thin cross sections of multi-stepped vibrating structures. From the view point of this theory the governing equation contains a mixed $x-t$ derivative of fourth order which leads to the appearance of a limiting point in the frequency spectrum of the system. Furthermore, the boundary and continuity conditions are modified to take into consideration the lateral inertia effects. From the view point of engineering applications the Rayleigh-Love theory is not difficult and it helps to substantially improve the accuracy of the frequency spectrum predictions in comparison with results based on the classical theory.

R. Bishop (Bishop, 1952) further modified the Rayleigh-Love theory in 1952 by taking into account the lateral shear effects. The theory is referred to as the Rayleigh-Bishop theory. The governing equation of this theory contains a fourth order $x$ derivative together with a mixed fourth order $x$ - $t$ derivative. This eliminates the limiting point of the frequency spectrum of the system, but adds an additional boundary condition on shear stress. An interesting aspect of the Rayleigh-Bishop theory is that, when applied to multi-stepped structures, it is necessary to guarantee zero shear continuity conditions at the junctions of neighbouring sections. From the view point of engineering applications this theory is slightly more complicated than the Rayleigh-Love theory. This theory predicts better resonance frequencies than the classical theory but is also limited by its application to low frequency vibrations.

The classical, Rayleigh-Love and Rayleigh-Bishop theories are based on the fundamental assumption of unimodality, which means that the dynamics of the rod is described by a single unknown function and hence, a single partial differential equation. Another fundamental assumption is that the above mentioned theories are plane cross sectional theories, i.e. displacements in the longitudinal direction preserve their plane and are not functions of distance from the neutral longitudinal axis of the rod. The boundary conditions on the outer cylindrical surface of the rod are fully ignored in the classical theory. In the Rayleigh-Love theory these boundary conditions are implicitly taken into consideration because the radial component of the surface stress is zero (and moreover it is always zero inside the rod) and the shear stress is neglected. In the Rayleigh-Bishop theory the radial component of the surface stress is zero inside and on the outer surface of the rod but the shear stress is taken to consideration inside the rod and hence, it is non-zero on the outer surface of the rod. Therefore these theories are limited to low frequency applications and cannot be used for description of high frequency effects such as the propagation of surface waves.

At higher excitation frequencies, the higher order modes of vibration can be activated and waves with the same frequencies but different wave number will propagate through the bar. The unimodal theories cannot be used to describe this phenomenon, which led to the development of the first multimodal theory by R. Mindlin and G. Herrmann in 1950, now referred to as the Mindlin-Herrmann theory (Graff, 1991:510-521). The Mindlin-Herrmann theory is a two modal plane cross sectional theory, where the lateral displacement mode is defined by a function that is independent of the longitudinal displacement mode of the rod. The dynamics of a rod modelled by the Mindlin-Herrmann theory is therefore described by a system of two coupled partial differential equation.

Investigation of the Pochhammer-Chree frequency equation shows that the frequency of the lateral mode depends on the Poisson ratio $\eta$ in such a way that the frequency increases with increasing $\eta$. When $\eta>0.2833$, the frequency of the lateral mode is higher than the frequency of the lowest axial shear mode, which is not dependent on $\eta$. This means that the Mindlin- 
Herrmann (two mode) theory is not well suited for analysis of higher frequency effects, due to the absence of the third (lowest axial shear) mode. This led to the development of a multimodal non plane cross sectional theory by R. Mindlin and H. McNiven in 1960, now referred to as the Mindlin-McNiven theory (Mindlin \& McNiven, 1960). In the MindlinMcNiven theory the number of modes is not restricted and every individual mode is proportional to a Jacobi function of radius of the rod. Mindlin and McNiven further considered a three mode "second order approximation" of their theory, in which the coupling of the longitudinal, lateral and lowest axial shear modes was analysed. The dependence of the frequency spectrum of the lateral mode on the Poisson ratio was also exposed. The main advantage of this approach is in the simplicity of the orthogonality conditions. The main drawback of the theory is that the property of the smallness of the cross section with respect to the length of the rod could not be explicitly formulated because the high order Jacobi functions contain terms linearly proportional to the radius of the rod. The trade-off between simplicity of the boundary conditions and clarity of the physical formulation of the problem was one of the most important features of the subsequent development of the theory of longitudinal vibrations of finite rods. It is important to mention that the boundary conditions on the outer cylindrical surface of the rod are ignored by both of Mindlin's theories.

Modern theory of longitudinal vibration of rods is developing in two different directions. In both cases, the displacement fields are expressed as a power series expansion with respect to the distance from the neutral longitudinal axis of the rod (the radial coordinate $r$ ).

The first branch is focussed on the development of unimodal non plane cross sectional theories where either the radial or shear stress boundary conditions (or both) on the outer cylindrical surface are taken into consideration. By equating either the radial or shear stress component to zero throughout the entire thickness (and hence, also on the outer surface) of the rod, all the lateral and axial shear modes can be defined in terms of the longitudinal mode and its derivatives. Hence, the unimodal theories are each described by a single partial differential equation in the longitudinal mode. This theory is taken as the basis for nonlinear generalisation of the linear effects for analysis of soliton propagation in solid rods (Porubov, 2003:66-72). The Rayleigh-Love and Rayleigh-Bishop models are also defined within the frames of this theory.

Second, the theory of longitudinal vibrations of the rod is generalised by means of creation of multimodal approaches where the longitudinal, lateral and axial shear modes are assumed to be independent of one another, as in the case the Mindlin-McNiven theory. In this case, it is possible to take into consideration radial and shear boundary conditions or part of them on the outer cylindrical surface of the rod. One of the recent attempts of development of this approach was presented as the Zachmanoglou-Volterra theory (Grigoljuk \& Selezov, 1973: 106-107; Zachmanoglou \& Volterra, 1958). This is a four mode non plane cross section theory where the fourth mode is specially matched so to satisfy the zero radial stress boundary condition on the outer cylindrical surface of the rod (the shear stress is non-zero in this theory). Hence, the dynamics of the rod is described by a system of three coupled partial differential equations.

In the above mentioned publications the authors considered mainly longitudinal wave propagation in infinite and semi-infinite rods. This paper will focus on the general methods of solution of the problem of longitudinal vibration of finite length rods for all of the above mentioned theories. The main approach is based on formulation of the equations of motion and boundary conditions using energy methods (Hamilton's principle), finding two 
orthogonality conditions (Fedotov et al., 2010) and obtaining the general solution of the problems in terms of Green functions. Most of the results obtained by the method of two orthogonalities for derivation of the Green functions are published in this paper for the first time.

The term multimodal theory has been introduced to describe theories in which the longitudinal and lateral displacements described by more than one independent function, where the number of modes is equivalent to the number of independent functions. Theories in which the longitudinal and lateral displacements are described by a single function have been given the term unimode theory. The terms plane cross sectional and non-plane cross sectional theories will also be used in this article. A plane cross sectional theory is based on the assumption that each point $x$ on the longitudinal axis of the rod represents a plane cross section of the bar (orthogonal to the $x$ axis) and that, during deformation, the plane cross sections remain plane. This assumption was first made during the derivation of the classical wave equation. In a non plane cross sectional theory, axial shear modes are introduced and each point $x$ no longer represent a plane cross section of the bar. For example, if the lowest axial shear mode is defined by the term $r^{2} u(x, t)$, then a point $x$, located on the $x$-axis, no longer represents displacement of a plane cross section, but rather that of a circular paraboloid section. This concept was first introduced by Mindlin and McNiven (Mindlin \& McNiven, 1960).

\section{Derivation of the system of equations of motion}

Consider a solid cylindrical bar with radius $R$ and length $l$ which experiences longitudinal vibration along the $x$-axis and lateral shear vibrations, transverse to the $x$-axis in the direction of the $r$-axis and in the tangential direction. Consider an axisymmetric problem and suppose that the axial and lateral wave displacements can be written as a power series expansion in the radial coordinate $r$ of the form

$$
u=u(x, r, t)=u_{0}(x, t)+r^{2} u_{2}(x, t)+\ldots+r^{2 m} u_{2 m}(x, t)
$$

and

$$
w=w(x, r, t)=r u_{1}(x, t)+r^{3} u_{3}(x, t)+\ldots+r^{2 n+1} u_{2 n+1}(x, t)
$$

respectively. The displacements in the tangential direction are assumed to be negligible $(v(x, r, \varphi, t)=0)$. That is, no torsional vibrations are present. The longitudinal and lateral displacements defined in (1)-(2) are similar to those proposed by Mindlin and McNiven. Mindlin and McNiven, however, represented the expansion of $u$ and $w$ in series of Jacobi polynomials in the radial coordinate $r$ (Mindlin \& McNiven, 1960). A representation based on the power series expansion in (1)-(2) was first introduced by Mindlin and Herrmann in 1950 to describe their two mode theory (Graff, 1991:510-521), and later by Zachmanoglou and Volterra to describe their four mode theory (Grigoljuk \& Selezov, 1973: 106-107; Zachmanoglou \& Volterra, 1958). According to choice of $m$ and $n$ in (1)-(2), different models of longitudinal vibration of bars can be obtained, including the well-known models such as those of Rayleigh-Love, Rayleigh-Bishop, Mindlin-Hermann and a three-mode model analogous to the Mindlin-McNiven "second order approximation".

The geometrical characteristics of deformation of the bar are defined by the following linear elastic strain tensor field: 


$$
\begin{array}{lll}
\varepsilon_{x x}=\partial_{x} u, & \varepsilon_{r r}=\partial_{r} w, & \varepsilon_{\varphi \varphi}=\frac{\partial_{\varphi} v}{r}+\frac{w}{r}=\frac{w}{r}, \\
\varepsilon_{x r}=\partial_{r} u+\partial_{x} w, & \varepsilon_{\varphi x}=\frac{\partial_{\varphi} u}{r}+\partial_{x} v=0, & \varepsilon_{\varphi r}=\partial_{r} v-\frac{v}{r}+\frac{\partial_{\varphi} w}{r}=0 .
\end{array}
$$

The compact notation $\partial_{\alpha}=\partial / \partial \alpha$ is used. Due to axial symmetry, it follows that $\varepsilon_{\varphi r}=\varepsilon_{\varphi x}=0$. The stress tensor due to the isotropic properties of the bar is calculated from Hook's Law as follows:

$$
\begin{aligned}
& \sigma_{x x}=(\lambda+2 \mu) \varepsilon_{x x}+\lambda\left(\varepsilon_{r r}+\varepsilon_{\varphi \varphi}\right), \\
& \sigma_{\varphi r}=\mu \varepsilon_{\varphi r}=0, \\
& \sigma_{r r}=(\lambda+2 \mu) \varepsilon_{r r}+\lambda\left(\varepsilon_{x x}+\varepsilon_{\varphi \varphi}\right), \\
& \sigma_{x r}=\mu \varepsilon_{x r} \\
& \sigma_{\varphi \varphi}=(\lambda+2 \mu) \varepsilon_{\varphi \varphi}+\lambda\left(\varepsilon_{x x}+\varepsilon_{r r}\right), \\
& \sigma_{\varphi x}=\mu \varepsilon_{\varphi x}=0 .
\end{aligned}
$$

$\lambda$ and $\mu$ are Lame's constants, defined as (Fung \& Tong, 2001:141)

$$
\mu=\frac{E}{2(1+\eta)} \text { and } \lambda=\frac{E \eta}{(1-2 \eta)(1+\eta)}
$$

where $\eta$ and $E$ are the Poisson ratio and Young's modulus of elasticity respectively.

The equations of motion (and associated boundary conditions) can be derived using Hamilton's variational principle. The Lagrangian is defined as $L=T-P$, where

$$
T=\frac{\rho}{2} \int_{0}^{l} \int_{s}\left(\dot{u}^{2}+\dot{w}^{2}\right) d s d x
$$

is the kinetic energy of the system, representing the energy yielded (supplied) by the displacement of the disturbances (vibrations),

$$
P=\frac{1}{2} \int_{0}^{l} \int_{s}\left(\sigma_{x x} \varepsilon_{x x}+\sigma_{r r} \varepsilon_{r r}+\sigma_{\varphi \varphi} \varepsilon_{\varphi \varphi}+\sigma_{x r} \varepsilon_{x r}\right) d s d x
$$

is the strain energy of the system, representing the potential energy stored in the bar by elastic straining, $S=\int_{S} d s=\int_{0}^{2 \pi} \int_{0}^{R} r d r d \varphi=\pi R^{2}$ is the cross sectional area and $\rho$ is the mass density of the rod.

Exact solutions of the boundary value (or mixed) problems resulting from the application of Hamilton's principle can be found using the method of separation of variables, by assuming that the solution can be written in the form of a generalised Fourier series

$$
u_{j}(x, t)=\sum_{m=1}^{\infty} y_{j m}(x) \Phi(t), \quad j=0,1,2, \ldots, n-1
$$

where the set of functions $\left\{y_{j m}(x)\right\}, j=0,1,2, \ldots, n-1$ are the eigenfunctions of the corresponding Sturm-Liouville problem and $n$ is the number of independent functions 
chosen to represent the longitudinal and lateral displacements in (1)-(2). It is possible to prove that the eigenfunctions satisfy two orthogonality conditions:

$$
\left(y_{m}, y_{n}\right)_{1}=\left\|y_{n}\right\|_{1}^{2} \delta_{n m} \text { and }\left(y_{m}, y_{n}\right)_{2}=\left\|y_{n}\right\|_{2}^{2} \delta_{n m}
$$

where $y_{m}$ refers to the set of eigenfunctions $\left\{y_{j m}(x)\right\}, j=0,1,2, \ldots, n-1$ corresponding to a particular eigenvalue $\omega_{m}$ and $\delta_{n m}$ is the Kronecker-Delta function. The unkown function in time $\Phi(t)$ can be found by substituting (8) into the Lagrangian of the system and making use of the orthogonality conditions (9).

\subsection{Unimodal theories}

The unimodal theories are derived by establishing a linear dependence of all the displacement modes in (1) and (2) on the first longitudinal displacement mode $u_{0}(x, t)$ and its derivates. This dependence is obtained by substituting (1) and (2) into the equations for the radial and axial shear stress components $\sigma_{r r}$ and $\sigma_{x r}$ and equating all terms at equal powers of the radial coordinate $r$ to zero. By introducing these constraints, either the radial stress (when an even number of terms are considered) or the axial shear stress (when an uneven number of terms are considered) will be equal to zero, not only on the free outer surface, but throughout the entire thickness of the rod. That is, at least one of the classical boundary conditions for the free outer surface of a multidimensional bar, $\sigma_{r r}=0$ and $\sigma_{x r}=0$ at $r=R$, will be satisfied.

It is further possible to artificially ensure that the remaining non-zero boundary condition term ( $\sigma_{r r}$ for an uneven number of terms and $\sigma_{x r}$ for an even number of terms) is also equal to zero throughout the entire thickness of the bar by neglecting its effect (that is, assuming this term is zero) when computing the potential energy function (7). This assumption was first introduced independently by both Rayleigh and Love in deriving the well known RayleighLove (two term, unimode) theory. For this reason, all unimode theories where this assumption is made will subsequently be referred to as Rayleigh-Love type theories. Unimode theories in which this assumption is not made will be referred to as RayleighBishop type theories.

Substituting the resulting kinetic and potential energy functions, given by (6) and (7), into the Lagrangian yields

$$
L=T-P=\int_{0}^{l} \Lambda\left(\dot{u}_{0}^{(j-1)}, u_{0}^{(j)}\right) d x, \quad j=1,2, \ldots, n
$$

where $\Lambda$ is known as the Lagrangian density and $n$ is number of terms chosen to represent longitudinal and lateral displacements in (1)-(2). The upper dot denotes the derivative with respect to time $t$ and $u_{0}^{(j)}$ is the $j^{\text {th }}$ derivative of $u_{0}(x, t)$ with respect to the axial coordinate $x$. Hamilton's Principle shows that the Lagrangian density $\Lambda$ satisfies a Euler-Lagrange partial differential equation (typically) of the form:

$$
\sum_{j=1}^{n}\left\{(-1)^{j-1} \frac{\partial^{j}}{\partial x^{j-1} \partial t}\left(\frac{\partial \Lambda}{\partial \dot{u}_{0}^{(j-1)}}\right)+(-1)^{j-1} \frac{\partial^{j}}{\partial x^{j}}\left(\frac{\partial \Lambda}{\partial u_{0}^{(j)}}\right)\right\}=0
$$

where $n$ is the number of dependent terms in (1)-(2). 


\section{a) The classical theory}

The classical theory is the simplest of the models discussed in this article. The longitudinal displacement is represented by

$$
u=u(x, t)=u_{0}(x, t)
$$

and lateral displacements are assumed negligible $(w=0)$. Since, for the classical model, $\eta=0$ (a fortiori $\lambda=0$ ) and $E=2 \mu$, the Lagrangian density of the system is given by

$$
\Lambda\left(\dot{u}_{0}, u_{0}^{\prime}\right)=\frac{1}{2}\left(\rho S \dot{u}_{0}^{2}-E S u_{0}^{\prime 2}\right)
$$

which satisfies the Euler-Lagrange differential equation

$$
\frac{\partial}{\partial t}\left(\frac{\partial \Lambda}{\partial \dot{u}_{0}}\right)+\frac{\partial}{\partial x}\left(\frac{\partial \Lambda}{\partial u_{0}^{\prime}}\right)=0
$$

The prime denotes the derivate with respect to the axial coordinate $x$. Substituting (13) into (14) leads to the familiar classical wave equation

$$
\rho \partial_{t}^{2} u_{0}-E \partial_{x}^{2} u_{0}=0
$$

The associated natural (free end) $\left.u_{0}^{\prime}(x, t)\right|_{x=0, l}=0$ or essential (fixed end) $\left.u_{0}(x, t)\right|_{x=0, l}=0$ boundary conditions are derived directly from Hamilton's principle.

The eigenfunctions $\left\{y_{0 n}(x)\right\}$ of the corresponding Sturm-Liouville problem satisfy the two orthogonality conditions (9) where

$$
\left(y_{m}, y_{n}\right)_{1}=\int_{0}^{l} y_{0 m}(x) y_{0 n}(x) d x, \quad\left(y_{m}, y_{n}\right)_{2}=\int_{0}^{l} y_{0 m}^{\prime}(x) y_{0 n}^{\prime}(x) d x
$$

\section{b) The Rayleigh-Bishop theory}

In the Rayleigh-Bishop theory, the longitudinal and lateral displacements are represented by the two term expansion

$$
\begin{aligned}
& u(x, t)=u_{0}(x, t) \\
& w(x, r, t)=r u_{1}(x, t)
\end{aligned}
$$

Substituting (17) into the equation for the radial stress component $\sigma_{r r}$ and equating all terms at $r^{0}$ to zero yields $u_{1}(x, t)=-\eta u_{0}^{\prime}(x, t)$. That is, the lateral displacement of a particle distant $r$ from the axis is assumed to be proportional to the longitudinal strain:

$$
w(x, r, t)=-\eta r \partial_{x} u(x, t)=-\eta r u_{0}^{\prime}(x, t)
$$

Applying this constraint to the lateral displacement means that $\sigma_{r r}=0$ throughout the entire thickness of the bar. The Rayleigh-Bishop model is categorised as a unimode, plane cross section model, since both longitudinal and lateral displacements are defined in terms of a single mode of displacement, $u_{0}$, and the term $-\eta r u_{0}^{\prime}(x, t)$ in (18) implies that lateral deformation occurs in plane and hence all plane cross sections remain plane during deformation. Substituting (17) and (18) into (6) and (7) results in 


$$
T=\frac{1}{2} \int_{0}^{l}\left(\rho S \dot{u}_{0}^{2}+\rho \eta I_{2} \dot{u}_{0}^{\prime 2}\right) d x
$$

and

$$
P=\frac{1}{2} \int_{0}^{l}\left(S E u_{0}^{\prime 2}+\mu \eta^{2} I_{2} u_{0}^{\prime \prime 2}\right) d x
$$

where $I_{2}=\int_{s} r^{2} d s=\pi R^{4} / 2$ is the polar moment of inertia of the cross section. The Lagrangian density of the system is given by

$$
\Lambda\left(\dot{u}_{0}, u_{0}^{\prime}, \dot{u}_{0}^{\prime}, u_{0}^{\prime \prime}\right)=\frac{1}{2}\left(\rho S \dot{u}_{0}^{2}+\rho \eta^{2} I_{2} \dot{u}_{0}^{\prime 2}-S E u_{0}^{\prime 2}-\eta^{2} \mu I_{2} u_{0}^{\prime \prime}\right)
$$

which satisfies the Euler-Lagrange differential equation

$$
\frac{\partial}{\partial t}\left(\frac{\partial \Lambda}{\partial \dot{u}_{0}}\right)+\frac{\partial}{\partial x}\left(\frac{\partial \Lambda}{\partial u_{0}^{\prime}}\right)-\frac{\partial^{2}}{\partial x \partial t}\left(\frac{\partial \Lambda}{\partial \dot{u}_{0}^{\prime}}\right)-\frac{\partial^{2}}{\partial x^{2}}\left(\frac{\partial \Lambda}{\partial u_{0}^{\prime \prime}}\right)=0
$$

The Rayleigh-Bishop equation (Gai et al., 2007) is thus obtained

$$
S\left(\rho \partial_{t}^{2} u_{0}-E \partial_{x}^{2} u_{0}\right)-\eta^{2} I_{2} \partial_{x}^{2}\left(\rho \partial_{t}^{2} u_{0}-\mu \partial_{x}^{2} u_{0}\right)=0
$$

A combination of the natural (free ends)

$$
\begin{aligned}
{\left.\left[S E u_{0}^{\prime}(x, t)+\rho \eta^{2} I_{2} \ddot{u}_{0}^{\prime}(x, t)-\eta^{2} I_{2} \mu u_{0}^{\prime \prime \prime}(x, t)\right]\right|_{x=0, l} } & =0, \quad \text { and } \\
\left.u_{0}^{\prime \prime}(x, t)\right|_{x=0, l} & =0
\end{aligned}
$$

or the essential (fixed ends)

$$
\left.u_{0}(x, t)\right|_{x=0, l}=0 \quad \text { and }\left.\quad u_{0}^{\prime}(x, t)\right|_{x=0, l}=0
$$

boundary conditions can be used at the end points $x=0$ and $x=l$.

It is possible to prove that the eigenfunctions $\left\{y_{0 n}(x)\right\}$ of the corresponding Sturm-Liouville problem satisfy the two orthogonality conditions (9) where

$$
\begin{aligned}
& \left(y_{m}, y_{n}\right)_{1}=\int_{0}^{l}\left[S y_{0 m}(x) y_{0 n}(x)+\eta^{2} I_{2} y_{0 m}^{\prime}(x) y_{0 n}^{\prime}(x)\right] d x \\
& \left(y_{m}, y_{n}\right)_{2}=\int_{0}^{l}\left[S E y_{0 m}^{\prime}(x) y_{0 n}^{\prime}(x)+\mu \eta^{2} I_{2} y_{0 m}^{\prime \prime}(x) y_{0 n}^{\prime \prime}(x)\right] d x
\end{aligned}
$$

\section{c) The Rayleigh-Love theory}

As in the case of the Rayleigh-Bishop theory, the longitudinal and lateral displacements for the Rayleigh-Love theory are defined by (17) and (18). It is clear that the Rayleigh-Love theory is a unimode, plane cross sectional theory, for the same reasons as discussed above for the Rayleigh-Bishop case. 
In contrast to the Rayleigh-Bishop theory, Rayleigh and Love made the additional assumption that only the inertial effect of the lateral displacements are taken into consideration and the effect of stiffness on shear stress is neglected when computing the potential energy function. That is, $\varepsilon_{x r}=\partial_{x} w \neq 0$ and $\sigma_{x r} \approx 0$. Under these assumptions, the kinetic energy function is given by (19) and the potential energy function is reduced to

$$
P=\frac{1}{2} \int_{0}^{l} S E u_{0}^{\prime 2} d x
$$

The Langrangian density of the system

$$
\Lambda\left(\dot{u}_{0}, u_{0}^{\prime}, \dot{u}_{0}^{\prime}\right)=\frac{1}{2}\left(\rho S \dot{u}_{0}^{2}+\rho \eta^{2} I_{2} \dot{u}_{0}^{\prime 2}-S E u_{0}^{\prime 2}\right)
$$

satisfies the Euler-Lagrange differential equation:

$$
\frac{\partial}{\partial t}\left(\frac{\partial \Lambda}{\partial \dot{u}_{0}}\right)+\frac{\partial}{\partial x}\left(\frac{\partial \Lambda}{\partial u_{0}^{\prime}}\right)-\frac{\partial^{2}}{\partial x \partial t}\left(\frac{\partial \Lambda}{\partial \dot{u}_{0}^{\prime}}\right)=0
$$

Substituting (28) into (29) leads to the Rayleigh-Love equation of motion (Fedotov et al., 2007):

$$
S\left(\rho \partial_{t}^{2} u_{0}-E \partial_{x}^{2} u_{0}\right)-\eta^{2} I_{2} \rho \partial_{x}^{2}\left(\partial_{t}^{2} u_{0}\right)=0
$$

A combination of the natural (free ends)

$$
\left.\left[S E u_{0}^{\prime}(x, t)+\rho \eta^{2} I_{2} \ddot{u}_{0}^{\prime}(x, t)\right]\right|_{x=0, l}=0
$$

or the essential (fixed ends)

$$
\left.u_{0}(x, t)\right|_{x=0, l}=0
$$

boundary conditions can be used at the end points $x=0$ and $x=l$. Neglecting the shear stress term $\sigma_{x r}$ in the potential energy function has resulted in the absence of the term with fourth order $x$ derivative in (23). This will result in a supremum (limit point) for the set of eigenvalues $\omega_{n}$. Note also that, due to the absence of the fourth order $x$ derivative, the number of boundary conditions at each end have been reduced from two to only one.

It is possible to prove that the eigenfunctions $\left\{y_{0 n}(x)\right\}$ of the corresponding Sturm-Liouville problem satisfy the two orthogonality conditions (9) where

$$
\begin{aligned}
& \left(y_{m}, y_{n}\right)_{1}=\int_{0}^{l}\left[S y_{0 m}(x) y_{0 n}(x)+\eta^{2} I_{2} y_{0 m}^{\prime}(x) y_{0 n}^{\prime}(x)\right] d x \\
& \left(y_{m}, y_{n}\right)_{2}=\int_{0}^{l} y_{0 m}^{\prime}(x) y_{0 n}^{\prime}(x) d x
\end{aligned}
$$

\section{d) Three term Rayleigh-Bishop type theory}

Consider the case where the longitudinal and lateral displacements are defined by the three term expansion 


$$
\begin{aligned}
& u(x, r, t)=u_{0}(x, t)+r^{2} u_{2}(x, t) \\
& w(x, r, t)=r u_{1}(x, t)
\end{aligned}
$$

This is a non plane cross sectional theory due to the presence of the axial shear term $r^{2} u_{2}(x, t)$. Substituting (34) into the equations for radial and axial shear stress and equating the terms at $r^{0}$ and $r^{1}$ yields

$$
u_{1}(x, t)=-\eta u_{0}^{\prime}(x, t) \quad \text { and } \quad u_{2}(x, t)=-\frac{1}{2} u_{1}^{\prime}(x, t)=\frac{\eta}{2} u_{0}^{\prime \prime}(x, t)
$$

It follows that the axial shear stress $\sigma_{x r}=0$ throughout the entire thickness of the bar. Making use of the relations $\lambda+2 \mu-2 \lambda \eta=E$ and $2 \eta(\lambda+\mu)=\lambda$, the Lagrangian density of the system can be represented as

$$
\begin{aligned}
\Lambda & =\Lambda\left(\dot{u}_{0}, u_{0}^{\prime}, \dot{u}_{0}^{\prime}, \dot{u}_{0}^{\prime \prime}, u_{0}^{\prime \prime \prime}\right) \\
& =\frac{1}{2}\left(\rho S \dot{u}_{0}^{2}+\rho \eta I_{2} \dot{u}_{0} \dot{u}_{0}^{\prime \prime}+\rho \frac{\eta^{2}}{4} I_{4} \dot{u}_{0}^{\prime \prime 2}+\rho \eta^{2} I_{2} \dot{u}_{0}^{\prime 2}-S E u_{0}^{\prime 2}-\eta I_{2} E u_{0}^{\prime} u_{0}^{\prime \prime \prime}-\frac{\eta^{2}}{4} I_{4}(\lambda+2 \mu) u_{0}^{\prime \prime \prime 2}\right)
\end{aligned}
$$

where $I_{4}=\int_{s} r^{4} d s=\pi R^{6} / 3$ is a property of the cross section of the rod. The Lagrangian density (36) satisfies the Euler-Lagrange differential equation

$$
\frac{\partial}{\partial t}\left(\frac{\partial \Lambda}{\partial \dot{u}_{0}}\right)+\frac{\partial}{\partial x}\left(\frac{\partial \Lambda}{\partial u_{0}^{\prime}}\right)-\frac{\partial^{2}}{\partial x \partial t}\left(\frac{\partial \Lambda}{\partial \dot{u}_{0}^{\prime}}\right)+\frac{\partial^{3}}{\partial x^{2} \partial t}\left(\frac{\partial \Lambda}{\partial \dot{u}_{0}^{\prime \prime}}\right)+\frac{\partial^{3}}{\partial x^{3}}\left(\frac{\partial \Lambda}{\partial u_{0}^{\prime \prime \prime}}\right)=0
$$

which leads to the equation of motion for the three term Rayleigh-Bishop type model

$$
S\left(\rho \ddot{u}_{0}-E u_{0}^{\prime \prime}\right)+\eta I_{2} \partial_{x}^{2}\left(\rho \ddot{u}_{0}-E u_{0}^{\prime \prime}\right)+\frac{\eta^{2}}{4} I_{4} \partial_{x}^{4}\left[\rho \ddot{u}_{0}-(\lambda+2 \mu) u_{0}^{\prime \prime}\right]-\rho \eta^{2} I_{2} \ddot{u}_{0}^{\prime \prime}=0
$$

with the corresponding natural (free ends)

$$
\begin{gathered}
{\left.\left[-S E u_{0}^{\prime}-\eta I_{2} E u_{0}^{\prime \prime \prime}-\rho \eta^{2} I_{2} \ddot{u}_{0}^{\prime}+\rho \frac{\eta}{2} I_{2} \ddot{u}_{0}^{\prime}+\rho \frac{\eta^{2}}{4} I_{4} \ddot{u}_{0}^{\prime \prime \prime}-\frac{\eta^{2}}{4} I_{4}(\lambda+2 \mu) u_{0}^{(\mathrm{v})}\right]\right|_{x=0, l}=0, \quad \text { and }} \\
{\left.\left[-\rho \frac{\eta}{2} I_{2} \ddot{u}_{0}-\rho \frac{\eta^{2}}{4} I_{4} \ddot{u}_{0}^{\prime \prime}+\frac{\eta}{2} I_{2} E u_{0}^{\prime \prime}+\frac{\eta^{2}}{4} I_{4}(\lambda+2 \mu) u_{0}^{(\mathrm{iv})}\right]\right|_{x=0, l}=0, \quad \text { and }} \\
{\left.\left[-\frac{\eta}{2} I_{2} E u_{0}^{\prime}-\frac{\eta^{2}}{4} I_{4}(\lambda+2 \mu) u_{0}^{\prime \prime \prime}\right]\right|_{x=0, l}=0 .}
\end{gathered}
$$

or essential (fixed ends)

$$
\left.u_{0}\right|_{x=0, l}=0 \text { and }\left.\quad u_{0}^{\prime}\right|_{x=0, l}=0 \text { and }\left.\quad u_{0}^{\prime \prime}\right|_{x=0, l}=0
$$

boundary conditions at the end points $x=0$ and $x=l$.

It is possible to prove that the eigenfunctions $\left\{y_{0 n}(x)\right\}$ of the corresponding Sturm-Liouville problem satisfy the two orthogonality conditions (9) where 


$$
\begin{aligned}
& \left(y_{m}, y_{n}\right)_{1}=\int_{0}^{l}\left(S y_{n} y_{m}+\frac{\eta}{2} I_{2} y_{n}^{\prime \prime} y_{m}+\frac{\eta}{2} I_{2} y_{n} y_{m}^{\prime \prime}+\frac{\eta^{2}}{4} I_{4} y_{n}^{\prime \prime} y_{m}^{\prime \prime}+\eta^{2} I_{2} y_{n}^{\prime} y_{m}^{\prime}\right) d x \\
& \left(y_{m}, y_{n}\right)_{2}=\int_{0}^{l}\left(S E y_{n}^{\prime} y_{m}^{\prime}+\frac{\eta}{2} I_{2} E y_{n}^{\prime \prime \prime} y_{m}^{\prime}+\frac{\eta}{2} I_{2} E y_{n}^{\prime} y_{m}^{\prime \prime \prime}+\frac{\eta^{2}}{4} I_{4}(\lambda+2 \mu) y_{n}^{\prime \prime \prime} y_{m}^{\prime \prime \prime}\right) d x
\end{aligned}
$$

\section{e) Three term Rayleigh-Love type theory}

As in the case of the three term Rayleigh-Bishop type theory, the longitudinal and lateral displacements for the three term Rayleigh-Love type theory are given by (34) and (35). In this theory, an additional Rayleigh-Love type assumption is made to neglect the radial stress component, which is non-zero in this case, when calculating the potential energy of the system. That is, $\varepsilon_{r r}=\partial_{r} w \neq 0$ and $\sigma_{r r} \approx 0$. Under these assumptions, the Lagrangian density of the system becomes

$$
\begin{aligned}
\Lambda & =\Lambda\left(\dot{u}_{0}, u_{0}^{\prime}, \dot{u}_{0}^{\prime}, \dot{u}_{0}^{\prime \prime}, u_{0}^{\prime \prime \prime}\right) \\
& =\frac{1}{2}\left[\rho S \dot{u}_{0}^{2}+\rho \eta^{2} I_{2} \dot{u}_{0}^{\prime 2}+\rho \eta I_{2} \dot{u}_{0} \dot{u}_{0}^{\prime \prime}+\rho \frac{\eta^{2}}{4} I_{4} \dot{u}_{0}^{\prime \prime 2}-S E u_{0}^{\prime 2}-\eta I_{2} E u_{0}^{\prime} u_{0}^{\prime \prime \prime}-\frac{\eta^{2}}{2} I_{2} \lambda u_{0}^{\prime} u_{0}^{\prime \prime \prime}-\right. \\
& \left.-\frac{\eta^{2}}{4} I_{4}(\lambda+2 \mu) u_{0}^{\prime \prime 2}\right]
\end{aligned}
$$

The Lagrangian density (42) satisfies the Euler-Lagrange differential equation

$$
\frac{\partial}{\partial t}\left(\frac{\partial \Lambda}{\partial \dot{u}_{0}}\right)+\frac{\partial}{\partial x}\left(\frac{\partial \Lambda}{\partial u_{0}^{\prime}}\right)-\frac{\partial^{2}}{\partial x \partial t}\left(\frac{\partial \Lambda}{\partial \dot{u}_{0}^{\prime}}\right)+\frac{\partial^{3}}{\partial x^{2} \partial t}\left(\frac{\partial \Lambda}{\partial \dot{u}_{0}^{\prime \prime}}\right)+\frac{\partial^{3}}{\partial x^{3}}\left(\frac{\partial \Lambda}{\partial u_{0}^{\prime \prime \prime}}\right)=0
$$

which leads to the equation of motion for the three term Rayleigh-Love type model:

$$
S\left(\rho \ddot{u}_{0}-E u_{0}^{\prime \prime}\right)+\eta I_{2} \partial_{x}^{2}\left(\rho \ddot{u}_{0}-E u_{0}^{\prime \prime}\right)+\frac{\eta^{2}}{4} I_{4} \partial_{x}^{4}\left[\rho \ddot{u}_{0}-(\lambda+2 \mu) u_{0}^{\prime \prime}\right]-\rho \eta^{2} I_{2} \ddot{u}_{0}^{\prime \prime}-\frac{\eta^{2}}{2} I_{2} \lambda \partial_{x}^{4}\left(u_{0}\right)=0
$$

with the corresponding natural (free ends)

$$
\begin{gathered}
{\left.\left[-S E u_{0}^{\prime}-\eta I_{2} E u_{0}^{\prime \prime \prime}-\frac{\eta^{2}}{2} I_{2} \lambda u_{0}^{\prime \prime \prime}-\rho \eta^{2} I_{2} \ddot{u}_{0}^{\prime}+\rho \frac{\eta}{2} I_{2} \ddot{u}_{0}^{\prime}+\rho \frac{\eta^{2}}{4} I_{4} \ddot{u}_{0}^{\prime \prime \prime}-\frac{\eta^{2}}{4} I_{4}(\lambda+2 \mu) u_{0}^{(\mathrm{v})}\right]\right|_{x=0, l}=0, \quad \text { and }} \\
{\left.\left[-\rho \frac{\eta}{2} I_{2} \ddot{u}_{0}-\rho \frac{\eta^{2}}{4} I_{4} \ddot{u}_{0}^{\prime \prime}+\frac{\eta}{2} I_{2} E u_{0}^{\prime \prime}+\frac{\eta^{2}}{4} I_{4}(\lambda+2 \mu) u_{0}^{(\mathrm{iv})}+\frac{\eta^{2}}{4} I_{2} \lambda u_{0}^{\prime \prime}\right]\right|_{x=0, l}=0, \quad \text { and }} \\
{\left.\left[-\frac{\eta}{2} I_{2} E u_{0}^{\prime}-\frac{\eta^{2}}{4} I_{4}(\lambda+2 \mu) u_{0}^{\prime \prime \prime}-\frac{\eta^{2}}{4} I_{2} \lambda u_{0}^{\prime}\right]\right|_{x=0, l}=0 .}
\end{gathered}
$$

or the essential (fixed ends)

$$
\left.u_{0}\right|_{x=0, l}=0 \text { and }\left.\quad u_{0}^{\prime}\right|_{x=0, l}=0 \text { and }\left.\quad u_{0}^{\prime \prime}\right|_{x=0, l}=0
$$

boundary conditions at the end points $x=0$ and $x=l$. It should be noted that in this case, the Rayleigh-Love type assumption that $\sigma_{r r}=0 \mathrm{did}$ not result in a simplification of the equation 
of motion or boundary conditions as was the case in the two term theories discussed above. This is true for all unimode theories in which an uneven number of terms have been considered to represent longitudinal and lateral displacements.

It is possible to prove that the eigenfunctions $\left\{y_{0 n}(x)\right\}$ of the corresponding Sturm-Liouville problem satisfy the two orthogonality conditions (9) where

$$
\begin{aligned}
\left(y_{m}, y_{n}\right)_{1}= & \int_{0}^{l}\left\{S y_{n} y_{m}+\frac{\eta}{2} I_{2} y_{n}^{\prime \prime} y_{m}+\frac{\eta}{2} I_{2} y_{n} y_{m}^{\prime \prime}+\frac{\eta^{2}}{4} I_{4} y_{n}^{\prime \prime} y_{m}^{\prime \prime}+\eta^{2} I_{2} y_{n}^{\prime} y_{m}^{\prime}\right\} d x \\
\left(y_{m}, y_{n}\right)_{2}= & \int_{0}^{l}\left\{S E y_{n}^{\prime} y_{m}^{\prime}+\frac{\eta}{2} I_{2} E y_{n}^{\prime \prime \prime} y_{m}^{\prime}+\frac{\eta}{2} I_{2} E y_{n}^{\prime} y_{m}^{\prime \prime \prime}+\frac{\eta^{2}}{4} I_{4}(\lambda+2 \mu) y_{n}^{\prime \prime \prime} y^{\prime \prime \prime}+\right. \\
& \left.+\frac{\eta^{2}}{4} I_{2} \lambda y_{n}^{\prime \prime \prime} y_{m}^{\prime}+\frac{\eta^{2}}{4} I_{2} \lambda y_{n}^{\prime} y_{m}^{\prime \prime \prime}\right\} d x
\end{aligned}
$$

\section{f) Four term Rayleigh-Bishop type theory}

Consider the case where the longitudinal and lateral displacements are defined by the three term expansion

$$
\begin{aligned}
& u(x, r, t)=u_{0}(x, t)+r^{2} u_{2}(x, t) \\
& w(x, r, t)=r u_{1}(x, t)+r^{3} u_{3}(x, t)
\end{aligned}
$$

Substituting (48) into the equations for radial and axial shear stress and equating the terms at $r^{0}, r^{1}$ and $r^{2}$ yields

$$
u_{1}=-\eta u_{0}^{\prime}, \quad u_{2}=-\frac{1}{2} u_{1}^{\prime}=\frac{\eta}{2} u_{0}^{\prime \prime} \quad \text { and } \quad u_{3}=-\frac{\eta}{3-2 \eta} u_{2}^{\prime}=-\frac{\eta^{2}}{2(3-2 \eta)} u_{0}^{\prime \prime \prime}
$$

It follows that the radial stress $\sigma_{r r}$ is zero throughout the entire thickness of the bar. Making use of the relations $\lambda+2 \mu-2 \lambda \eta=E, 2 \eta(\lambda+\mu)=\lambda$ and $2 \mu \eta=\lambda(1-2 \eta)$, the Lagrangian density of the system can be given by

$$
\begin{aligned}
& \Lambda=\frac{1}{2}\left[\rho S \dot{u}_{0}^{2}+\rho \eta I_{2} \dot{u}_{0} \dot{u}_{0}^{\prime \prime}+\rho \frac{\eta^{2}}{4} I_{4} \dot{u}_{0}^{\prime \prime 2}+\rho \eta^{2} I_{2} \dot{u}_{0}^{\prime 2}+\rho \frac{\eta^{3}}{(3-2 \eta)} I_{4} \dot{u}_{0}^{\prime} \dot{u}_{0}^{\prime \prime \prime}+\rho \frac{\eta^{4}}{4(3-2 \eta)^{2}} I_{6} \dot{u}_{0}^{\prime \prime \prime 2}-\right. \\
& -S E u_{0}^{\prime 2}-\eta I_{2} E u_{0}^{\prime} u_{0}^{\prime \prime \prime}-\frac{\eta^{2}}{4} I_{4}(\lambda+2 \mu) u_{0}^{\prime \prime 2}-\frac{\eta^{4}}{4(3-2 \eta)^{2}} I_{6} \mu\left(u_{0}^{(\mathrm{iv})}\right)^{2}+\frac{\eta^{3}}{(3-2 \eta)} I_{4} \lambda u_{0}^{\prime \prime 2}+ \\
& \left.+\frac{\eta^{4}}{(3-2 \eta)^{2}} I_{4} \mu u_{0}^{\prime \prime \prime 2}\right]
\end{aligned}
$$

where $I_{6}=\int_{s} r^{6} d s=\pi R^{8} / 4$ is a property of the cross section of the rod. The Langrangian density of the system satisfies the Euler-Lagrange partial differential equation

$\frac{\partial}{\partial t}\left(\frac{\partial \Lambda}{\partial \dot{u}_{0}}\right)+\frac{\partial}{\partial x}\left(\frac{\partial \Lambda}{\partial u_{0}^{\prime}}\right)-\frac{\partial^{2}}{\partial x \partial t}\left(\frac{\partial \Lambda}{\partial \dot{u}_{0}^{\prime}}\right)+\frac{\partial^{3}}{\partial x^{2} \partial t}\left(\frac{\partial \Lambda}{\partial \dot{u}_{0}^{\prime \prime}}\right)+\frac{\partial^{3}}{\partial x^{3}}\left(\frac{\partial \Lambda}{\partial u_{0}^{\prime \prime \prime}}\right)-\frac{\partial^{4}}{\partial x^{3} \partial t}\left(\frac{\partial \Lambda}{\partial \dot{u}_{0}^{\prime \prime \prime}}\right)-\frac{\partial^{4}}{\partial x^{4}}\left(\frac{\partial \Lambda}{\partial u_{0}^{(\mathrm{iv})}}\right)=0$ 
which leads to the equation of motion for the four term Rayleigh-Bishop type model

$$
\begin{aligned}
& S\left(\rho \ddot{u}_{0}-E u_{0}^{\prime \prime}\right)+\eta I_{2} \partial_{x}^{2}\left(\rho \ddot{u}_{0}-E u_{0}^{\prime \prime}\right)+\frac{\eta^{2}}{4} I_{4} \partial_{x}^{4}\left[\rho \ddot{u}_{0}-(\lambda+2 \mu) u_{0}^{\prime \prime}\right]-\frac{\eta^{4}}{4(3-2 \eta)^{2}} I_{6} \partial_{x}^{6}\left(\rho \ddot{u}_{0}-\mu u_{0}^{\prime \prime}\right)- \\
& -\rho \eta^{2} I_{2} \ddot{u}_{0}^{\prime \prime}-\frac{\eta^{3}}{(3-2 \eta)} I_{4} \partial_{x}^{4}\left(\rho \ddot{u}_{0}\right)+\frac{\eta^{3}}{(3-2 \eta)} I_{4} \partial_{x}^{6}\left(\lambda u_{0}\right)+\frac{\eta^{4}}{(3-2 \eta)^{2}} I_{4} \partial_{x}^{6}\left(\mu u_{0}\right)=0
\end{aligned}
$$

with the corresponding natural (free ends)

$$
\begin{gathered}
{\left.\left[\frac{\partial \Lambda}{\partial u_{0}^{\prime}}-\frac{\partial}{\partial t}\left(\frac{\partial \Lambda}{\partial \dot{u}_{0}^{\prime}}\right)+\frac{\partial^{2}}{\partial x \partial t}\left(\frac{\partial \Lambda}{\partial \dot{u}_{0}^{\prime \prime}}\right)+\frac{\partial^{2}}{\partial x^{2}}\left(\frac{\partial \Lambda}{\partial u_{0}^{\prime \prime \prime}}\right)-\frac{\partial^{3}}{\partial x^{2} \partial t}\left(\frac{\partial \Lambda}{\partial \dot{u}_{0}^{\prime \prime}}\right)-\frac{\partial^{3}}{\partial x^{3}}\left(\frac{\partial \Lambda}{\partial u_{0}^{(\mathrm{iv})}}\right)\right]\right|_{x=0, l}=0 \quad \text { and }} \\
{\left.\left[-\frac{\partial}{\partial t}\left(\frac{\partial \Lambda}{\partial \dot{u}_{0}^{\prime \prime}}\right)-\frac{\partial}{\partial x}\left(\frac{\partial \Lambda}{\partial u_{0}^{\prime \prime \prime}}\right)+\frac{\partial^{2}}{\partial x \partial t}\left(\frac{\partial \Lambda}{\partial \dot{u}_{0}^{\prime \prime \prime}}\right)+\frac{\partial^{2}}{\partial x^{2}}\left(\frac{\partial \Lambda}{\partial u_{0}^{(\mathrm{iv})}}\right)\right]\right|_{x=0, l}=0 \text { and }} \\
{\left.\left[\frac{\partial \Lambda}{\partial u_{0}^{\prime \prime \prime}}-\frac{\partial}{\partial t}\left(\frac{\partial \Lambda}{\partial \dot{u}_{0}^{\prime \prime \prime}}\right)-\frac{\partial}{\partial x}\left(\frac{\partial \Lambda}{\partial u_{0}^{(\mathrm{iv})}}\right)\right]\right|_{\left.\right|_{x=0, l}}=0 \text { and }} \\
{\left.\left[\frac{\partial \Lambda}{\partial u_{0}^{(\mathrm{iv})}}\right]\right|_{x=0, l}=0}
\end{gathered}
$$

or essential (fixed ends)

$$
\left.u_{0}\right|_{x=0, l}=0 \quad \text { and }\left.\quad u_{0}^{\prime}\right|_{x=0, l}=0 \text { and }\left.\quad u_{0}^{\prime \prime}\right|_{x=0, l}=0 \text { and }\left.\quad u_{0}^{\prime \prime \prime}\right|_{x=0, l}=0
$$

boundary conditions at the end points $x=0$ and $x=l$. The explicit form of the boundary conditions (53) can be determined using the Lagrangian density (50).

\section{g) Four term Rayleigh-Love type theory}

As in the case of the three term Rayleigh-Bishop type theory, the longitudinal and lateral displacements for this theory are given by (48) and (49). The constraints (49) result in $\sigma_{r r}=0$ and $\sigma_{x r} \neq 0$ throughout the entire thickness of the bar. An additional Rayleigh-Love type assumption is made to neglect the axial shear stress component when calculating the potential energy of the system. Under these assumptions, the Lagrangian density of the system reduces to

$$
\begin{aligned}
\Lambda= & \frac{1}{2}\left[\rho S \dot{u}_{0}^{2}+\rho \eta I_{2} \dot{u}_{0} \dot{u}_{0}^{\prime \prime}+\rho \frac{\eta^{2}}{4} I_{4} \dot{u}_{0}^{\prime \prime 2}+\rho \eta^{2} I_{2} \dot{u}_{0}^{\prime 2}+\rho \frac{\eta^{3}}{(3-2 \eta)} I_{4} \dot{u}_{0}^{\prime} \dot{u}_{0}^{\prime \prime \prime}+\rho \frac{\eta^{4}}{4(3-2 \eta)^{2}} I_{6} \dot{u}_{0}^{\prime \prime 2}-\right. \\
& \left.-S E u_{0}^{\prime 2}-\eta I_{2} E u_{0}^{\prime} u_{0}^{\prime \prime \prime}-\frac{\eta^{2}}{4} I_{4}(\lambda+2 \mu) u_{0}^{\prime \prime \prime 2}+\frac{\eta^{3}}{(3-2 \eta)} I_{4} \lambda u_{0}^{\prime \prime 2}+\frac{\eta^{4}}{(3-2 \eta)^{2}} I_{4} \mu u_{0}^{\prime \prime \prime 2}\right]
\end{aligned}
$$

The Langrangian density of the system satisfies the Euler-Lagrange differential equation

$$
\frac{\partial}{\partial t}\left(\frac{\partial \Lambda}{\partial \dot{u}_{0}}\right)+\frac{\partial}{\partial x}\left(\frac{\partial \Lambda}{\partial u_{0}^{\prime}}\right)-\frac{\partial^{2}}{\partial x \partial t}\left(\frac{\partial \Lambda}{\partial \dot{u}_{0}^{\prime}}\right)+\frac{\partial^{3}}{\partial x^{2} \partial t}\left(\frac{\partial \Lambda}{\partial \dot{u}_{0}^{\prime \prime}}\right)+\frac{\partial^{3}}{\partial x^{3}}\left(\frac{\partial \Lambda}{\partial u_{0}^{\prime \prime \prime}}\right)-\frac{\partial^{4}}{\partial x^{3} \partial t}\left(\frac{\partial \Lambda}{\partial \dot{u}_{0}^{\prime \prime \prime}}\right)=0
$$


which leads to the equation of motion for the three mode Rayleigh-Love type model:

$$
\begin{gathered}
S\left(\rho \ddot{u}_{0}-E u_{0}^{\prime \prime}\right)+\eta I_{2} \partial_{x}^{2}\left(\rho \ddot{u}_{0}-E u_{0}^{\prime \prime}\right)+\frac{\eta^{2}}{4} I_{4} \partial_{x}^{4}\left[\rho \ddot{u}_{0}-(\lambda+2 \mu) u_{0}^{\prime \prime}\right]-\frac{\eta^{4}}{4(3-2 \eta)^{2}} I_{6} \partial_{x}^{6}\left(\rho \ddot{u}_{0}\right)- \\
-\rho \eta^{2} I_{2} \ddot{u}_{0}^{\prime \prime}-\frac{\eta^{3}}{(3-2 \eta)} I_{4} \partial_{x}^{4}\left(\rho \ddot{u}_{0}\right)+\frac{\eta^{3}}{(3-2 \eta)} I_{4} \partial_{x}^{6}\left(\lambda u_{0}\right)+\frac{\eta^{4}}{(3-2 \eta)^{2}} I_{4} \partial_{x}^{6}\left(\mu u_{0}\right)=0
\end{gathered}
$$

A combination of the natural (free ends)

$$
\begin{gathered}
{\left.\left[\frac{\partial \Lambda}{\partial u_{0}^{\prime}}-\frac{\partial}{\partial t}\left(\frac{\partial \Lambda}{\partial \dot{u}_{0}^{\prime}}\right)+\frac{\partial^{2}}{\partial x \partial t}\left(\frac{\partial \Lambda}{\partial \dot{u}_{0}^{\prime \prime}}\right)+\frac{\partial^{2}}{\partial x^{2}}\left(\frac{\partial \Lambda}{\partial u_{0}^{\prime \prime \prime}}\right)-\frac{\partial^{3}}{\partial x^{2} \partial t}\left(\frac{\partial \Lambda}{\partial \dot{u}_{0}^{\prime \prime \prime}}\right)\right]\right|_{x=0, l}=0 \text { and }} \\
{\left.\left[-\frac{\partial}{\partial t}\left(\frac{\partial \Lambda}{\partial \dot{u}_{0}^{\prime \prime}}\right)-\frac{\partial}{\partial x}\left(\frac{\partial \Lambda}{\partial u_{0}^{\prime \prime \prime}}\right)+\frac{\partial^{2}}{\partial x \partial t}\left(\frac{\partial \Lambda}{\partial \dot{u}_{0}^{\prime \prime \prime}}\right)\right]\right|_{x=0, l}=0 \text { and }} \\
{\left.\left[\frac{\partial \Lambda}{\partial u_{0}^{\prime \prime \prime}}-\frac{\partial}{\partial t}\left(\frac{\partial \Lambda}{\partial \dot{u}_{0}^{\prime \prime \prime}}\right)\right]\right|_{x=0, l}=0}
\end{gathered}
$$

or the essential (fixed ends)

$$
\left.u_{0}\right|_{x=0, l}=0, \quad \text { and }\left.\quad u_{0}^{\prime}\right|_{x=0, l}=0, \quad \text { and }\left.\quad u_{0}^{\prime \prime}\right|_{x=0, l}=0 .
$$

boundary conditions can be used at the end points $x=0$ and $x=l$. The explicit form of the boundary conditions (58) can be determined from the Lagrangian density (55). Once again, neglecting the shear stress term $\sigma_{x r}$ in the potential energy function has simplified the equation of motion and boundary conditions. The highest order $x$ derivative (in this case, eighth order) does not appear in the equation of motion (57) and the number of boundary conditions at each end has been reduced from four to three. The absence of the eighth order $x$ derivative, together with the presence of the mixed eighth order $x$ - $t$ derivate will also result in a limit point for the set of eigenvalues $\omega_{n}$. This simplification of the equation of motion and boundary conditions is true for all unimode theories in which an even number of terms have been considered to represent longitudinal and lateral displacements.

\subsection{Multimodal theories}

The multimodal theories are derived from the representation of longitudinal and lateral displacements (1)-(2), where all the terms are assumed to be independent of one another. Substituting the resulting kinetic and potential energy functions, given by (6) and (7), into the Lagrangian yields

$$
L=T-P=\int_{0}^{l} \Lambda\left(u_{j}, \dot{u}_{j}, u_{j}^{\prime}\right) d x
$$

where $j$ depends on the choice of $n$ and $m$ in (1)-(2). Hamilton's Principle shows that the Lagrangian density $\Lambda$ satisfies a system of Euler-Lagrange differential equations (typically) of the form: 


$$
\begin{aligned}
& \frac{\partial}{\partial t}\left(\frac{\partial \Lambda}{\partial \dot{u}_{0}}\right)+\frac{\partial}{\partial x}\left(\frac{\partial \Lambda}{\partial u_{0}^{\prime}}\right)=0 \\
& \frac{\partial}{\partial t}\left(\frac{\partial \Lambda}{\partial \dot{u}_{j}}\right)+\frac{\partial}{\partial x}\left(\frac{\partial \Lambda}{\partial u_{j}^{\prime}}\right)-\frac{\partial \Lambda}{\partial u_{j}}=0, \quad j=1,2, \ldots, n-1
\end{aligned}
$$

where $n$ is the number of displacement modes (independent functions) chosen in (1)-(2). The upper dot and prime denote derivatives with respect to time $t$ and axial coordinate $x$ respectively.

\section{a) Two mode (Mindlin-Herrmann) theory}

In the case of the Mindlin-Herrmann theory, the longitudinal and lateral wave displacements are defined as

$$
\begin{aligned}
& u(x, t)=u_{0}(x, t) \\
& w(x, r, t)=r u_{1}(x, t)
\end{aligned}
$$

The Mindlin-Herrmann theory is the first (and the simplest) of the "multimode" theories, since two independent modes of displacement, $u_{0}(x, t)$ and $u_{1}(x, t)$, have been considered. The Mindlin-Herrmann theory is also a plane cross section theory, since the term $r u_{1}(x, t)$ in (62) implies that all plane cross sections remain plane during lateral (and longitudinal) deformation. It should be noted that both the Rayleigh-Love and Rayleigh-Bishop theories are special cases of the Mindlin-Herrmann theory and can be obtained from the MindlinHerrmann theory by introducing a constraint of the form $u_{1}+\eta u_{0}^{\prime}=0$ (that is, a constrained extremum).

Substituting (62) into (6) and (7) results in the following Lagrangian density of the system

$$
\begin{aligned}
\Lambda & =\Lambda\left(\dot{u}_{0}, \dot{u}_{1}, u_{0}^{\prime}, u_{1}^{\prime}, u_{1}\right) \\
& =\frac{1}{2}\left[\rho S \dot{u}_{0}^{2}+\rho I_{2} \dot{u}_{1}^{2}-S(\lambda+2 \mu) u_{0}^{\prime 2}-4 S \lambda u_{0}^{\prime} u_{1}-4 S(\lambda+\mu) u_{1}^{2}-I_{2} \mu u_{1}^{\prime 2}\right]
\end{aligned}
$$

which satisfies the following system of Euler-Lagrange differential equations:

$$
\begin{aligned}
& \frac{\partial}{\partial t}\left(\frac{\partial \Lambda}{\partial \dot{u}_{0}}\right)+\frac{\partial}{\partial x}\left(\frac{\partial \Lambda}{\partial u_{0}^{\prime}}\right)=0 \\
& \frac{\partial}{\partial t}\left(\frac{\partial \Lambda}{\partial \dot{u}_{1}}\right)+\frac{\partial}{\partial x}\left(\frac{\partial \Lambda}{\partial u_{1}^{\prime}}\right)-\frac{\partial \Lambda}{\partial u_{1}}=0
\end{aligned}
$$

Substituting (63) into (64) leads to the system of equations of motion:

$$
\begin{aligned}
& S\left[\rho \partial_{t}^{2} u_{0}-(\lambda+2 \mu) \partial_{x}^{2} u_{0}\right]-2 \lambda S \partial_{x} u_{1}=0 \\
& 2 \lambda S \partial_{x} u_{0}+I_{2}\left(\rho \partial_{t}^{2} u_{1}-\mu \partial_{x}^{2} u_{1}\right)+4 S(\lambda+\mu) u_{1}=0
\end{aligned}
$$

A combination of the natural

$$
\left.\left[(\lambda+2 \mu) u_{0}^{\prime}(x, t)+2 \lambda u_{1}(x, t)\right]\right|_{x=0, l}=0, \quad \text { and }\left.\quad u_{1}^{\prime}(x, t)\right|_{x=0, l}=0
$$


or the essential

$$
\left.u_{0}(x, t)\right|_{x=0, l}=0 \quad \text { and }\left.\quad u_{1}(x, t)\right|_{x=0, l}=0
$$

boundary conditions can be used at the end points $x=0$ and $x=l$.

It is possible to prove that the eigenfunctions $\left\{y_{0 n}(x)\right\}$ and $\left\{y_{1 n}(x)\right\}$ of the corresponding Sturm-Liouville problem satisfy the two orthogonality conditions (9) where

$$
\begin{aligned}
\left(y_{m}, y_{n}\right)_{1}= & \int_{0}^{l}\left[S y_{0 m}(x) y_{0 n}(x)+I_{2} y_{1 m}(x) y_{1 n}(x)\right] d x \\
\left(y_{m}, y_{n}\right)_{2}=\int_{0}^{l}\left[4 S(\lambda+\mu) y_{1 m}(x) y_{1 n}(x)+S(\lambda+2 \mu) y_{0 m}^{\prime}(x) y_{0 n}^{\prime}(x)+\right. & \\
& \left.+I_{2} \mu y_{1 m}^{\prime}(x) y_{1 n}^{\prime}(x)+2 S \lambda\left(y_{0 m}^{\prime}(x) y_{1 n}(x)+y_{0 n}^{\prime}(x) y_{1 m}(x)\right)\right] d x
\end{aligned}
$$

\section{b) Three mode theory}

Consider the case where the longitudinal and lateral displacements are defined by three modes of displacement as follows:

$$
\begin{aligned}
& u(x, r, t)=u_{0}(x, t)+r^{2} u_{2}(x, t) \\
& w(x, r, t)=r u_{1}(x, t)
\end{aligned}
$$

The longitudinal and lateral displacements defined in (69) are similar to those proposed by Mindlin and McNiven as a "second approximation" of their general theory.

The Lagrangian density of the system is given by

$$
\begin{aligned}
\Lambda= & \Lambda\left(\dot{u}_{0}, \dot{u}_{1}, \dot{u}_{2}, u_{0}^{\prime}, u_{1}^{\prime}, u_{2}^{\prime}, u_{1}, u_{2}\right) \\
= & \frac{1}{2}\left[\left(S \rho \dot{u}_{0}^{2}+2 I_{2} \rho \dot{u}_{0} \dot{u}_{2}+I_{2} \rho \dot{u}_{1}^{2}+I_{4} \rho \dot{u}_{2}^{2}\right)-(\lambda+2 \mu) S u_{0}^{\prime 2}-\mu I_{2} u_{1}^{\prime 2}-(\lambda+2 \mu) I_{4} u_{2}^{\prime 2}-\right. \\
& \left.-4 \lambda S u_{0}^{\prime} u_{1}-4 \mu I_{2} u_{1}^{\prime} u_{2}-2(\lambda+2 \mu) I_{2} u_{0}^{\prime} u_{2}^{\prime}-4 \lambda I_{2} u_{2}^{\prime} u_{1}-4(\lambda+\mu) S u_{1}^{2}-4 \mu I_{2} u_{2}^{2}\right]
\end{aligned}
$$

which satisfies the following system of Euler-Lagrange differential equations

$$
\begin{aligned}
& \frac{\partial}{\partial t}\left(\frac{\partial \Lambda}{\partial \dot{u}_{0}}\right)+\frac{\partial}{\partial x}\left(\frac{\partial \Lambda}{\partial u_{0}^{\prime}}\right)=0 \\
& \frac{\partial}{\partial t}\left(\frac{\partial \Lambda}{\partial \dot{u}_{1}}\right)+\frac{\partial}{\partial x}\left(\frac{\partial \Lambda}{\partial u_{1}^{\prime}}\right)-\frac{\partial \Lambda}{\partial u_{1}}=0 \\
& \frac{\partial}{\partial t}\left(\frac{\partial \Lambda}{\partial \dot{u}_{2}}\right)+\frac{\partial}{\partial x}\left(\frac{\partial \Lambda}{\partial u_{2}^{\prime}}\right)-\frac{\partial \Lambda}{\partial u_{2}}=0
\end{aligned}
$$

Substituting (70) into (71) yields the system of equations of motion:

$$
\begin{aligned}
& S\left[\rho \partial_{t}^{2} u_{0}-(\lambda+2 \mu) \partial_{x}^{2} u_{0}\right]-2 \lambda S \partial_{x} u_{1}+I_{2}\left[\rho \partial_{t}^{2} u_{2}-(\lambda+2 \mu) \partial_{x}^{2} u_{2}\right]=0 \\
& 2 \lambda S \partial_{x} u_{0}+I_{2}\left(\rho \partial_{t}^{2} u_{1}-\mu \partial_{x}^{2} u_{1}\right)+4 S(\lambda+\mu) u_{1}+2(\lambda-\mu) I_{2} \partial_{x} u_{2}=0 \\
& I_{2}\left[\rho \partial_{t}^{2} u_{0}-(\lambda+2 \mu) \partial_{x}^{2} u_{0}\right]-2(\lambda-\mu) I_{2} \partial_{x} u_{1}+I_{4}\left[\rho \partial_{t}^{2} u_{2}-(\lambda+2 \mu) \partial_{x}^{2} u_{2}\right]+4 \mu I_{2} u_{2}=0
\end{aligned}
$$


A combination of the natural

$$
\begin{aligned}
& {\left[S(\lambda+2 \mu) u_{0}^{\prime}(x, t)+2 S \lambda u_{1}(x, t)+\left.I_{2}(\lambda+2 \mu) u_{2}^{\prime}(x, t)\right|_{x=0, l}=0, \quad\right. \text { and }} \\
& {\left[I_{2} \mu u_{1}^{\prime}(x, t)+\left.2 I_{2} \mu u_{2}(x, t)\right|_{x=0, l}=0 \quad\right. \text { and }} \\
& {\left[I_{2}(\lambda+2 \mu) u_{0}^{\prime}(x, t)+2 I_{2} \lambda u_{1}(x, t)+I_{4}(\lambda+2 \mu) u_{2}^{\prime}(x, t)\right]_{x=0, l}=0}
\end{aligned}
$$

or essential

$$
\left.u_{0}(x, t)\right|_{x=0, l}=0 \quad \text { and }\left.\quad u_{1}(x, t)\right|_{x=0, l}=0 \quad \text { and }\left.\quad u_{2}(x, t)\right|_{x=0, l}=0
$$

boundary conditions can be used at the end points $x=0$ and $x=l$.

It is possible to prove that the eigenfunctions $\left\{y_{0 n}(x)\right\},\left\{y_{1 n}(x)\right\}$ and $\left\{y_{2 n}(x)\right\}$ of the corresponding Sturm-Liouville problem satisfy the two orthogonality conditions (9) where

$$
\begin{aligned}
&\left(y_{m}, y_{n}\right)_{1}=\int_{0}^{l}\left[S y_{0 m}(x) y_{0 n}(x)+I_{2} y_{1 m}(x) y_{1 n}(x)+I_{4} y_{2 m}(x) y_{2 n}(x)+\right. \\
&\left.+I_{2}\left(y_{0 m}(x) y_{2 n}(x)+y_{0 n}(x) y_{2 m}(x)\right)\right] d x \\
&\left(y_{m}, y_{n}\right)_{2}=\int_{0}^{l}\left[4 S(\lambda+\mu) y_{1 m}(x) y_{1 n}(x)+4 I_{2} \mu y_{2 m}(x) y_{2 n}(x)+S(\lambda+2 \mu) y_{0 m}^{\prime}(x) y_{0 n}^{\prime}(x)+\right. \\
&+ I_{2} \mu y_{1 m}^{\prime}(x) y_{1 n}^{\prime}(x)+I_{4}(\lambda+2 \mu) y_{2 m}^{\prime}(x) y_{2 n}^{\prime}(x)+ \\
&+2 S \lambda\left(y_{0 m}^{\prime}(x) y_{1 n}(x)+y_{0 n}^{\prime}(x) y_{1 m}(x)\right)+I_{2}(\lambda+2 \mu)\left(y_{0 m}^{\prime}(x) y_{2 n}^{\prime}(x)+y_{0 n}^{\prime}(x) y_{2 m}^{\prime}(x)\right)+ \\
&\left.+2 I_{2} \lambda\left(y_{1 m}(x) y_{2 n}^{\prime}(x)+y_{1 n}(x) y_{2 m}^{\prime}(x)\right)+2 I_{2} \mu\left(y_{1 m}^{\prime}(x) y_{2 n}(x)+y_{1 n}^{\prime}(x) y_{2 m}(x)\right)\right] d x
\end{aligned}
$$

\section{c) Four mode theory}

Consider the case where the longitudinal and lateral displacements are defined by four modes of displacement as follows

$$
\begin{aligned}
& u(x, r, t)=u_{0}(x, t)+r^{2} u_{2}(x, t) \\
& w(x, r, t)=r u_{1}(x, t)+r^{3} u_{3}(x, t)
\end{aligned}
$$

In a similar fashion as was described for the three mode theory above, the system of equations resulting from (76) may be written as

$$
\begin{aligned}
& S\left[\rho \partial_{t}^{2} u_{0}-(\lambda+2 \mu) \partial_{x}^{2} u_{0}\right]-d_{12} u_{1}+I_{2}\left[\rho \partial_{t}^{2} u_{2}-(\lambda+2 \mu) \partial_{x}^{2} u_{2}\right]-d_{14} u_{3}=0 \\
& d_{12} u_{0}+I_{2}\left(\rho \partial_{t}^{2} u_{1}-\mu \partial_{x}^{2} u_{1}\right)+b_{22} u_{1}+d_{23} u_{2}+I_{4}\left(\rho \partial_{t}^{2} u_{3}-\mu \partial_{x}^{2} u_{3}\right)+b_{24} u_{3}=0 \\
& I_{2}\left[\rho \partial_{t}^{2} u_{0}-(\lambda+2 \mu) \partial_{x}^{2} u_{0}\right]-d_{23} u_{1}+I_{4}\left[\rho \partial_{t}^{2} u_{2}-(\lambda+2 \mu) \partial_{x}^{2} u_{2}\right]+b_{33} u_{2}-d_{34} u_{3}=0 \\
& d_{14} u_{0}+I_{4}\left(\rho \partial_{t}^{2} u_{1}-\mu \partial_{x}^{2} u_{1}\right)+b_{24} u_{1}+d_{34} u_{2}+I_{6}\left(\rho \partial_{t}^{2} u_{3}-\mu \partial_{x}^{2} u_{3}\right)+b_{44} u_{3}=0
\end{aligned}
$$

where $d_{12}=2 \lambda \partial S_{x}, d_{14}=4 \lambda I_{2} \partial_{x}, d_{23}=2 I_{2}(\lambda-\mu) \partial_{x}$ and $d_{34}=2 I_{4}(2 \lambda-\mu) \partial_{x}$ are first order differential operators and $b_{22}=4 \mathrm{~S}(\lambda+\mu), b_{33}=4 I_{2} \mu, b_{24}=8 I_{2}(\lambda+\mu)$ and $b_{44}=4 I_{4}(4 \lambda+5 \mu)$ are numbers depending on $\lambda, \mu, S, I_{2}$ and $I_{4}$.

A combination of the natural 


$$
\begin{aligned}
{\left.\left[S(\lambda+2 \mu) u_{0}^{\prime}(x, t)+2 S \lambda u_{1}(x, t)+I_{2}(\lambda+2 \mu) u_{2}^{\prime}(x, t)+4 I_{2} \lambda u_{3}(x, t)\right]\right|_{x=0, l}=0, } & \text { and } \\
{\left.\left[I_{2} \mu u_{1}^{\prime}(x, t)+2 I_{2} \mu u_{2}(x, t)+I_{4} \mu u_{3}^{\prime}(x, t)\right]\right|_{x=0, l}=0, } & \text { and } \\
{\left.\left[I_{2}(\lambda+2 \mu) u_{0}^{\prime}(x, t)+2 I_{2} \lambda u_{1}(x, t)+I_{4}(\lambda+2 \mu) u_{2}^{\prime}(x, t)+4 I_{4} \lambda u_{3}(x, t)\right]\right|_{x=0, l}=0, } & \text { and } \\
{\left.\left[I_{4} \mu u_{1}^{\prime}(x, t)+2 I_{4} \mu u_{2}(x, t)+I_{6} \mu u_{3}^{\prime}(x, t)\right]\right|_{x=0, l}=0, } &
\end{aligned}
$$

or essential

$$
\left.u_{0}(x, t)\right|_{x=0, l}=0 \quad \text { and }\left.\quad u_{1}(x, t)\right|_{x=0, l}=0 \quad \text { and }\left.\quad u_{2}(x, t)\right|_{x=0, l}=0 \quad \text { and }\left.\quad u_{3}(x, t)\right|_{x=0, l}=0
$$

boundary conditions can be used at the end points $x=0$ and $x=l$.

\section{d) Zachmanoglou-Volterra theory}

In the Zachmanoglou-Volterra theory, the longitudinal and lateral displacements are defined by four modes of displacement as

$$
u=u_{0}(x, t)+r^{2} u_{2}(x, t)
$$

and

$$
w=r u_{1}(x, t)+r^{3} u_{3}(x, t)
$$

Zachmanoglou and Volterra also considered the additional condition that the radial stress component must be zero on the outer cylindrical surface of the bar, $\sigma_{r r}=0$ at $r=R$. From this condition, it follows that

$$
u_{3}(x, t)=\frac{1}{R^{2}(2 \eta-3)}\left[u_{1}(x, t)+\eta\left(u_{0}^{\prime}+R^{2} u_{2}^{\prime}\right)\right]
$$

That is, $u_{3}(x, t)$ is defined in terms of $u_{0}(x, t), u_{1}(x, t)$ and $u_{2}(x, t)$, and so the number of independent modes describing the vibration dynamics for the Zachmanoglou-Volterra theory has been reduced from four to three. The reduction of independent displacement modes results in a simplification of the four mode theory, without having an impact on model accuracy. The Lagrangian density of the system is given by

$$
\begin{aligned}
\Lambda & =\Lambda\left(\dot{u}_{0}, \dot{u}_{1}, \dot{u}_{2}, \dot{u}_{0}^{\prime}, \dot{u}_{2}^{\prime}, u_{0}^{\prime}, u_{1}^{\prime}, u_{2}^{\prime}, u_{0}^{\prime \prime}, u_{2}^{\prime \prime}, u_{1}, u_{2}\right) \\
& =\frac{1}{2}\left\{S \rho \dot{u}_{0}^{2}+I_{2} \rho \dot{u}_{1}^{2}+2 I_{2} \rho \dot{u}_{0} \dot{u}_{2}+I_{4} \rho \dot{u}_{2}^{2}+\frac{2 I_{4} \rho}{R^{2}(2 \eta-3)}\left(\dot{u}_{1}^{2}+\eta \dot{u}_{0}^{\prime} \dot{u}_{1}+R^{2} \eta \dot{u}_{1} \dot{u}_{2}^{\prime}\right)+\right. \\
& +\frac{I_{6} \rho}{R^{4}(2 \eta-3)^{2}}\left(\dot{u}_{1}^{2}+2 \eta \dot{u}_{0}^{\prime} \dot{u}_{1}+\eta^{2} \dot{u}_{0}^{\prime 2}+2 R^{2} \eta \dot{u}_{1} \dot{u}_{2}^{\prime}+2 R^{2} \eta^{2} \dot{u}_{0}^{\prime} \dot{u}_{2}^{\prime}+R^{4} \eta^{2} \dot{u}_{2}^{\prime 2}\right)- \\
& -S(\lambda+2 \mu) u_{0}^{\prime 2}-I_{2} \mu u_{1}^{\prime 2}-2 I_{2}(\lambda+2 \mu) u_{0}^{\prime} u_{2}^{\prime}-I_{4}(\lambda+2 \mu) u_{2}^{\prime 2}-\frac{2 I_{4}}{R^{2}(2 \eta-3)}\left(\mu u_{1}^{\prime 2}+\right. \\
& \left.+\eta \mu u_{0}^{\prime \prime} u_{1}^{\prime}+R^{2} \eta \mu u_{1}^{\prime} u_{2}^{\prime \prime}\right)-\frac{I_{6}}{R^{4}(2 \eta-3)^{2}}\left(\mu u_{1}^{\prime 2}+2 \eta \mu u_{0}^{\prime \prime} u_{1}^{\prime}+\eta^{2} \mu u_{0}^{\prime 2}+2 R^{2} \eta \mu u_{1}^{\prime} u_{2}^{\prime \prime}+\right.
\end{aligned}
$$




$$
\begin{aligned}
& \left.+2 R^{2} \eta^{2} \mu u_{0}^{\prime \prime} u_{2}^{\prime \prime}+R^{4} \eta^{2} \mu u_{2}^{\prime \prime 2}\right)-4 S(\lambda+\mu) u_{1}^{2}-4 S \lambda u_{0}^{\prime} u_{1}-4 I_{2} \mu u_{2}^{2}-4 I_{2} \lambda u_{1} u_{2}^{\prime}- \\
& -4 I_{2} \mu u_{1}^{\prime} u_{2}-\frac{I_{2}}{R^{2}(2 \eta-3)}\left[16(\lambda+\mu) u_{1}^{2}+8 \eta \lambda u_{0}^{\prime 2}+16 \lambda u_{0}^{\prime} u_{1}+8 R^{2} \eta \lambda u_{0}^{\prime} u_{2}^{\prime}+\right. \\
& \left.+8 R^{2} \lambda u_{1} u_{2}^{\prime}\right]-\frac{I_{4}}{R^{4}(2 \eta-3)^{2}}\left[8 \eta(4 \lambda+5 \mu) u_{0}^{\prime} u_{1}+4 \eta^{2}(4 \lambda+5 \mu) u_{0}^{\prime 2}-8 R^{2} \eta \mu u_{1} u_{2}^{\prime}-\right. \\
& \left.-8 R^{2} \eta^{2} \mu u_{0}^{\prime} u_{2}^{\prime}+4 R^{4} \eta^{2}(4 \lambda+5 \mu) u_{2}^{\prime 2}+4(4 \lambda+5 \mu) u_{1}^{2}-\right] \\
& \left.-\frac{I_{4}}{R^{2}(2 \eta-3)}\left(8 R^{2} \eta \lambda u_{2}^{\prime 2}+4 \eta \mu u_{0}^{\prime \prime} u_{2}+4 R^{2} \eta \mu u_{2} u_{2}^{\prime \prime}+4 \mu u_{1}^{\prime} u_{2}\right)\right\}
\end{aligned}
$$

The Lagrangian density satisfies the system of three Euler-Lagrange differential equations

$$
\begin{aligned}
& \frac{\partial}{\partial t}\left(\frac{\partial \Lambda}{\partial \dot{u}_{0}}\right)+\frac{\partial}{\partial x}\left(\frac{\partial \Lambda}{\partial u_{0}^{\prime}}\right)-\frac{\partial^{2}}{\partial x \partial t}\left(\frac{\partial \Lambda}{\partial \dot{u}_{0}^{\prime}}\right)-\frac{\partial^{2}}{\partial x^{2}}\left(\frac{\partial \Lambda}{\partial u_{0}^{\prime \prime}}\right)=0 \\
& \frac{\partial}{\partial t}\left(\frac{\partial \Lambda}{\partial \dot{u}_{1}}\right)+\frac{\partial}{\partial x}\left(\frac{\partial \Lambda}{\partial u_{1}^{\prime}}\right)-\frac{\partial \Lambda}{\partial u_{1}}=0 \\
& \frac{\partial}{\partial t}\left(\frac{\partial \Lambda}{\partial \dot{u}_{2}}\right)+\frac{\partial}{\partial x}\left(\frac{\partial \Lambda}{\partial u_{2}^{\prime}}\right)-\frac{\partial^{2}}{\partial x \partial t}\left(\frac{\partial \Lambda}{\partial \dot{u}_{2}^{\prime}}\right)-\frac{\partial^{2}}{\partial x^{2}}\left(\frac{\partial \Lambda}{\partial u_{2}^{\prime \prime}}\right)-\frac{\partial \Lambda}{\partial u_{2}}=0
\end{aligned}
$$

with the corresponding the natural

$$
\begin{aligned}
& {\left.\left[-\frac{\partial \Lambda}{\partial u_{0}^{\prime}}-\frac{\partial}{\partial t}\left(\frac{\partial \Lambda}{\partial \dot{u}_{0}^{\prime}}\right)-\frac{\partial}{\partial x}\left(\frac{\partial \Lambda}{\partial u_{0}^{\prime \prime}}\right)\right]\right|_{x=0, l}=0, \text { and }\left.\left[\frac{\partial \Lambda}{\partial u_{0}^{\prime \prime}}\right]\right|_{x=0, l}=0, \text { and }} \\
& {\left.\left[\frac{\partial \Lambda}{\partial u_{1}^{\prime}}\right]\right|_{x=0, l}=0, \text { and }} \\
& {\left.\left[-\frac{\partial \Lambda}{\partial u_{2}^{\prime}}-\frac{\partial}{\partial t}\left(\frac{\partial \Lambda}{\partial \dot{u}_{2}^{\prime}}\right)-\frac{\partial}{\partial x}\left(\frac{\partial \Lambda}{\partial u_{2}^{\prime \prime}}\right)\right]\right|_{x=0, l}=0, \text { and }\left.\left[\frac{\partial \Lambda}{\partial u_{2}^{\prime \prime}}\right]\right|_{x=0, l}=0}
\end{aligned}
$$

or essential

$$
\left.u_{0}\right|_{x=0, l}=0 \quad \text { and }\left.\quad u_{0}^{\prime}\right|_{x=0, l}=0 \quad \text { and }\left.\quad u_{1}\right|_{x=0, l}=0 \quad \text { and }\left.\quad u_{2}\right|_{x=0, l}=0 \quad \text { and }\left.\quad u_{2}^{\prime}\right|_{x=0, l}=0
$$

boundary conditions at the end points $x=0$ and $x=l$. The explicit form of the equations of motion and boundary conditions (84)-(85) can be determined from the Lagrangian density (83).

\section{Exact solution of the two mode (Mindlin-Herrmann) problem}

In what follows, the solution of one of the models considered in this article, namely that of the mixed Mindlin-Herrmann problem (65), (66), with initial conditions given by

$$
\begin{array}{ll}
\left.u_{0}(x, t)\right|_{t=0}=g(x), & \left.\dot{u}_{0}(x, t)\right|_{t=0}=h(x) \\
\left.u_{1}(x, t)\right|_{t=0}=\varphi(x), & \left.\dot{u}_{1}(x, t)\right|_{t=0}=q(x)
\end{array}
$$


is presented. Note that the boundary conditions (66) represent a bar with both ends free (natural boundary conditions).

Applying the method of eigenfunction orthogonalities for vibration problems (Fedotov et al., 2010) to problem (65), (66), (87), two types of orthogonality conditions are proved for the eigenfunctions. Assume the solution of the system (65) is of the form

$$
u_{0}(x, t)=y_{0}(x) e^{i w t} \quad u_{1}(x, t)=y_{1}(x) e^{i w t}
$$

where $i^{2}=-1$. After substituting (88) into (65) and the boundary conditions (66) the following Sturm-Liouville problem is obtained

$$
\begin{aligned}
& S\left[-\omega^{2} \rho y_{0}-(\lambda+2 \mu) \frac{d^{2}}{d x^{2}} y_{0}\right]-2 \lambda S \frac{d}{d x} y_{1}=0 \\
& 2 \lambda S \frac{d}{d x} y_{0}+I_{2}\left(-\omega^{2} \rho y_{1}-\mu \frac{d^{2}}{d x^{2}} y_{1}\right)+4 S(\lambda+\mu) y_{1}=0
\end{aligned}
$$

with the corresponding boundary conditions. Let $\left\{y_{0 m}(x)\right\}$ and $\left\{y_{1 m}(x)\right\}$ be the eigenfunctions of the Sturm-Liouville problem (89), which satisfy the two orthogonality conditions given by (9) and (68). The solution of the problem (65), (66), (87) can therefore be written as

$$
u_{0}(x, t)=S \int_{0}^{l}\left[g(\xi) \frac{\partial G_{1}(x, \xi, t)}{\partial t}+h(\xi) G_{1}(x, \xi, t)\right] d \xi+I_{2} \int_{0}^{l}\left[\varphi(\xi) \frac{\partial G_{2}(x, \xi, t)}{\partial t}+q(\xi) G_{2}(x, \xi, t)\right] d \xi
$$

and

$$
u_{1}(x, t)=S \int_{0}^{l}\left[g(\xi) \frac{\partial G_{3}(x, \xi, t)}{\partial t}+h(\xi) G_{3}(x, \xi, t)\right] d \xi+I_{2} \int_{0}^{l}\left[\varphi(\xi) \frac{\partial G_{4}(x, \xi, t)}{\partial t}+q(\xi) G_{4}(x, \xi, t)\right] d \xi
$$

where

$$
\begin{array}{ll}
G_{1}(x, \xi, t)=\sum_{n=1}^{\infty}\left(\frac{y_{0 n}(x) y_{0 n}(\xi) \sin \omega_{n} t}{\omega_{n}\left\|y_{n}\right\|_{1}^{2}}\right) & G_{2}(x, \xi, t)=\sum_{n=1}^{\infty}\left(\frac{y_{0 n}(x) y_{1 n}(\xi) \sin \omega_{n} t}{\omega_{n}\left\|y_{n}\right\|_{1}^{2}}\right) \\
G_{3}(x, \xi, t)=\sum_{n=1}^{\infty}\left(\frac{y_{1 n}(x) y_{0 n}(\xi) \sin \omega_{n} t}{\omega_{n}\left\|y_{n}\right\|_{1}^{2}}\right) & G_{4}(x, \xi, t)=\sum_{n=1}^{\infty}\left(\frac{y_{1 n}(x) y_{1 n}(\xi) \sin \omega_{n} t}{\omega_{n}\left\|y_{n}\right\|_{1}^{2}}\right)
\end{array}
$$

are the Green functions, and

$$
\omega_{n}=\frac{\left\|y_{n}\right\|_{2}}{\sqrt{\rho}\left\|y_{n}\right\|_{1}}, \quad n=1,2, \ldots
$$

are the eigenvalues (eigenfrequencies) of the problem. The solution of all other problems presented in this article can be obtained in a similar manner. The solution of the three mode problem, for example, can be obtained with six Green functions. 


\section{Predicting the accuracy of the approximate theories}

Two forms of graphical display are typically used to analyse the factors governing wave propagation for mathematical models describing the vibration of continuous systems. These are called the frequency spectrum and phase velocity dispersion curves and are obtained from the so-called frequency equation (Achenbach, 2005:206, 217-218; Graff, 1991:54), which shows the relation between frequency $\omega$, wave number $k$ and phase velocity $c$ for a particular model. In the " $k-\omega$ " plane the frequency equation for each model yields a number of continuous curves, called branches. The number of branches corresponds to the number of independent functions chosen to represent $u$ and $w$ in (1)-(2). Each branch shows the relationship between frequency $\omega$ and wave number $k$ for a particular mode of propagation. The collection of branches plotted in the " $k-\omega$ " plane is called the frequency spectrum of the system. Dispersion curves represent phase velocity $c$ versus wave number $k$ and can be obtained from the frequency equation by using the relation $\omega=c k$.

The different approximate models of longitudinal vibrations of rods can be analysed and deductions can be made regarding their accuracy by plotting their frequency spectra (or dispersion curves) and comparing them with the frequency spectrum (or dispersion curve) of the exact Pochhammer-Chree frequency equation for the axisymmetric problem of a cylindrical rod with free outer surface (longitudinal modes of vibration).

In order to find the frequency equation, it is assumed that each independent function can be represented as $u_{j}(x, t)=U_{j} e^{i(k x-\omega t)}$, where $j=0,1,2 \ldots n-1$ and $n$ corresponds to the number of independent functions chosen in (1)-(2). These representations for $u_{j}(x, t)$ are substituted into the equation(s) of motion, yielding the frequency equation. The frequency equation thus obtained for the classical theory is given by

$$
-\omega^{2}+c_{0}^{2} k^{2}=0
$$

which gives a single straight line with gradient $c_{0}=\sqrt{E} / \rho$, the speed of propagation of waves in an infinite rod described by the classical wave equation. The frequency equations for the Rayleigh-Love and Rayleigh-Bishop theories are given by

$$
-\omega^{2} S+k^{2} c_{0}^{2} S-\omega^{2} k^{2} \eta^{2} I_{2}=0
$$

and

$$
-\omega^{2} S+k^{2} c_{0}^{2} S-\omega^{2} k^{2} \eta^{2} I_{2}+k^{4} \eta^{2} I_{2} c_{2}^{2}=0
$$

respectively, where $c_{2}=\sqrt{\mu / \rho}$ is the speed of propagation of shear waves in an infinite rod. Since the classical, Rayleigh-Love and Rayleigh-Bishop theories are unimodal theories, their frequency equations yield a single branch in the " $k-\omega$ " domain That is, the frequency equations (92), (93) and (94) have a single solution for positive $\omega$. The Rayleigh-Love and Rayleigh-Bishop theories, however, do not yield straight lines as in the classical theory. That is, the Rayleigh-Love and Rayleigh-Bishop theories represent dispersive systems (the phase velocity $c$ depends on the wave number $k$ ). For the multimodal theories, the substitution results in a system of equations with unknowns $U_{j}$. The frequency equation is found by equating the determinant of the coefficient matrix to zero. The frequency equation for the Mindlin-Herrmann (two mode) theory, for example, can be thus obtained as 


$$
\omega^{4} I_{2}-\omega^{2} k^{2} I_{2}\left(c_{1}^{2}+c_{2}^{2}\right)-4 S\left(\omega^{2}-c_{0}^{2} k^{2}\right)\left(c_{1}^{2}-c_{2}^{2}\right)+k^{4} c_{1}^{2} c_{2}^{2} I_{2}=0
$$

where $c_{1}=\sqrt{(\lambda+2 \mu) / \rho}$ is the speed of propagation of pressure (dilatational) waves in an infinite rod. The well known Pochhammer-Chree frequency equation (Achenbach, 2005:242246; Graff, 1991:464-473) is given by

$$
\frac{2 \alpha}{R}\left(\beta^{2}+k^{2}\right) J_{1}(\alpha R) J_{1}(\beta R)-\left(\beta^{2}-k^{2}\right) J_{0}(\alpha R) J_{1}(\beta R)-4 k^{2} \alpha \beta J_{1}(\alpha R) J_{1}(\beta R)=0
$$

where $J_{n}(x)$ is the Bessel function of the first kind of order $n$,

$$
\alpha^{2}=\frac{\omega^{2}}{c_{1}^{2}}-k^{2}, \quad \beta^{2}=\frac{\omega^{2}}{c_{2}^{2}}-k^{2},
$$

and $R$ is the outer radius of the cylinder. The Pochhammer-Chree frequency equation yields infinitely many branches the " $k-\omega$ " and " $k-c$ " planes.

The figures that follow in this section have been generated for a cylindrical rod made from an Aluminium alloy with Young's modulus $E=70 \mathrm{GPa}$, mass density $\rho=2700 \mathrm{~kg} \cdot \mathrm{m}^{-3}$, and Poisson ratio $\eta=0.33$. It is not necessary to define the radius $R$ of the cylinder, since all frequency spectra and dispersion curves have been generated using the normalized, dimensionless parameters

$$
\Omega=\frac{\omega R}{\pi c_{2}}, \quad \bar{c}=\frac{c}{c_{0}}, \quad \xi=\frac{k R}{\pi}
$$

that are independent of the choice of $R$.

Figure 1a shows the frequency spectra for the classical, Rayleigh-Love and Rayleigh-Bishop theories, as well as the first branch of the two mode theory and the first branch of the exact Pochhammer-Chree frequency spectrum.

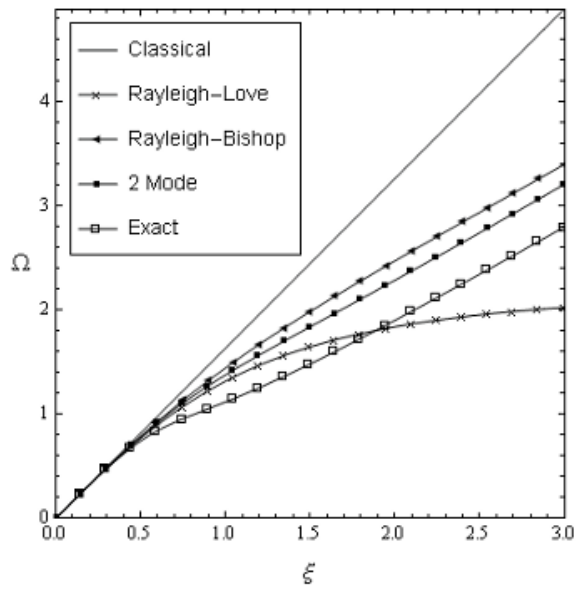

a)

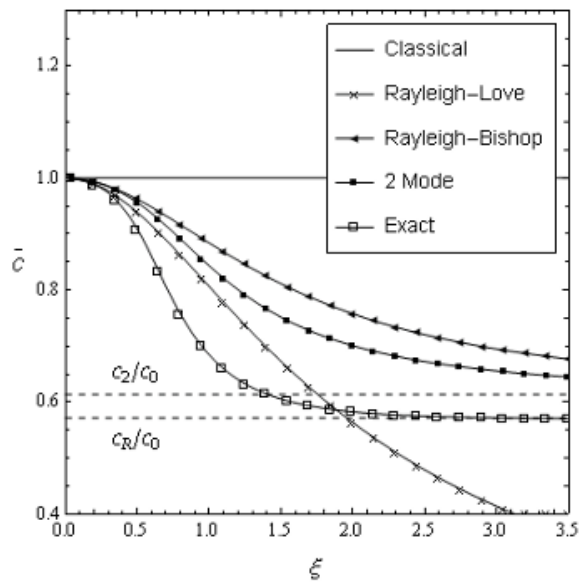

b)

Fig. 1. Comparison of a) frequency spectra and b) dispersion curves of the classical, Rayleigh-Love, Rayleigh-Bishop, two mode (first branch) and exact (first branch) theories. 
All the theories (including classical theory) approximately describe the first branch of the exact solution in a restricted " $k-\omega$ " domain. The Rayleigh-Love approximation is initially more accurate than the Rayleigh-Bishop and Mindlin-Herrmann approximations, but the values fall away rapidly for values of $\xi$ greater than 2 (approximately). The Rayleigh-Bishop and Mindlin-Herrmann approximations are reasonably accurate over a larger " $k-\omega$ "domain, but the branches asymptotically tend toward the shear wave solution, while the exact solution tends to the (Rayleigh) surface waves mode. The shear wave mode is given by the straight line $\omega(k)=c_{2} k$ and the surface wave mode is given by the straight line $\omega(k)=c_{\mathrm{R}} k$, where $c_{R} \approx 0.9320 c_{2}$ is the speed of propagation of surface waves in the rod. The factor 0.9320 is dependent on the Poisson ratio $\eta=0.33$ (Achenbach, 2005:187-194; Graff, 1991:323-328). The phenomenon described above is illustrated in figure $1 \mathrm{~b}$, which shows the phase velocity dispersion curves for the first branches of the exact solution and the Mindlin-Herrmann approximation, as well as the phase velocity dispersion curves for the Rayleigh-Love and Rayleigh-Bishop approximations.

The frequency spectra for some of the higher order unimode theories are given in figures $2 \mathrm{a}$ (Rayleigh-Bishop type theories) and $2 \mathrm{~b}$ (Rayleigh-Love type theories), together with the first branch of the exact Pochhammer-Chree frequency equation.

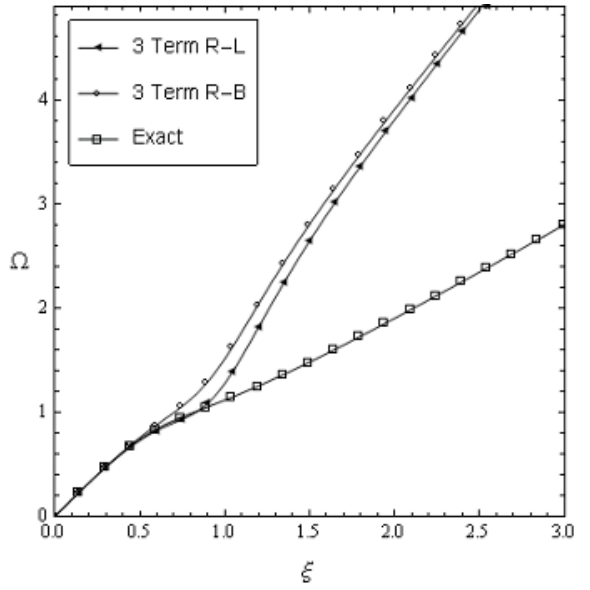

a)

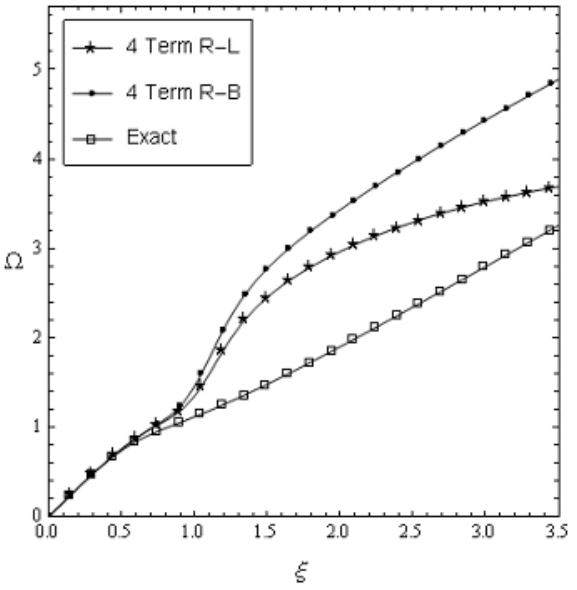

b)

Fig. 2. Frequency spectra of a) three term and b) four term R-L and R-B type theories.

The frequency spectra of the higher order unimode theories show unusual behaviour in the " $k-\omega$ " plane, due to the introduction of the higher order $x$ and mixed $x$ - $t$ derivative terms. The three term Rayleigh-Love and Rayleigh-Bishop type theories both tend towards the pressure waves mode $\omega(k)=c_{1} k$. The dispersion curve for this theory will show that $\bar{c}$ initially decreases with increasing $\xi$, but will then increase asymptotically to the velocity of pressure waves in an infinite bar. This is a property of all unimode theories where an uneven number of terms have been considered. The four term Rayleigh-Bishop type theory tends towards the shear wave solution. It is evident from the shape of the frequency spectrum that $\bar{c}$ will initially decrease with increasing $\xi$, but will then increase again before decreasing to the horizontal asymptote $c_{2} / c_{0}$. All Rayleigh-Bishop type theories where an even number of terms has been considered will tend towards the shear waves mode. The 
four term Rayleigh-Love type model, as discussed in section 3.1g above, has a limit point in its frequency spectrum, and the phase velocities of waves described by this theory will tend to zero as $\xi \rightarrow \infty$. The higher order unimode theories have been applied successfully in the analysis of propagation of solitons (solitary waves) in non-linear elastic solids. However, since the higher order theories are substantially more complicated than the (two term) Rayleigh-Love and Rayleigh-Bishop theories and due to the lack of physical clarity of the higher order derivative terms present in the equations of motion and boundary conditions, these theories are of little value for analysis of wave propagation in linear elastic solids.

Frequency spectra for a selection of multimode theories are presented in figures 3 through 7 . The exact Pochhammer-Chree frequency spectrum is shown by dashed lines on all the frequency spectrum plots shown for the multimode models. The imaginary branches (imaginary $\xi$ ) represent evanescent waves and describe exponential decay with respect to wave number. Figure 3 shows the frequency spectrum of the two mode (MindlinHerrmann) theory described in section 3.2a.

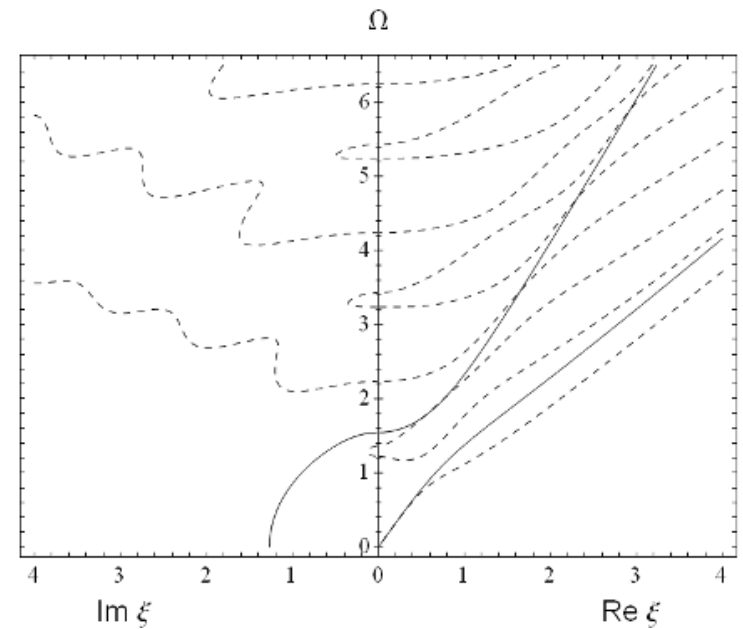

Fig. 3. Frequency spectrum of a two mode model (solid line), exact solution (dashed line).

Figure 4 shows the frequency spectrum of the three mode theory discussed in section $3.2 \mathrm{~b}$. Figure 5 shows the frequency spectrum of the four mode theory discussed in section 3.2c. Figure 6 shows the frequency spectrum of a five mode theory with three longitudinal and two lateral modes. Figure 7 shows the frequency spectrum of the Zachmanoglou-Volterra three mode theory discussed in section $3.2 \mathrm{~d}$.

It is apparent from analysis of the curves that the branches of the multimode theories approach those of the exact solution with increasing number of modes. That is, the higher the order of the multimode approximation (the greater the number of independent functions) the broader is the " $k$ - $\omega$ "- domain in which the effects of longitudinal vibrations of the rods could be analysed. To offset the error introduced by the omission of the higher order modes, Mindlin \& McNiven (1960) modified the kinetic and potential energy functions (in their three mode "second order approximation") by introducing four compensating factors that were chosen in such a way as to match the behaviour of the first three branches of the Pochhammer-Chree frequency spectrum at long wavelengths. This 
methodology could also be applied to the models presented here to obtain similar results. It should be noted that, regardless of the number of independent functions chosen, some of the branches will tend towards the shear wave solution $\omega(k)=c_{2} k$, while the remaining branches will tend towards the pressure wave solution $\omega(k)=c_{1} k$ as $k \rightarrow \infty$. None of the branches will tend towards the surface waves mode. This is because all the approximate theories discussed in this article are one dimensional theories (since $u_{k}=u_{k}(x, t)$ ), and can therefore not predict the effect of vibration on the outer cylindrical surface of the rod.

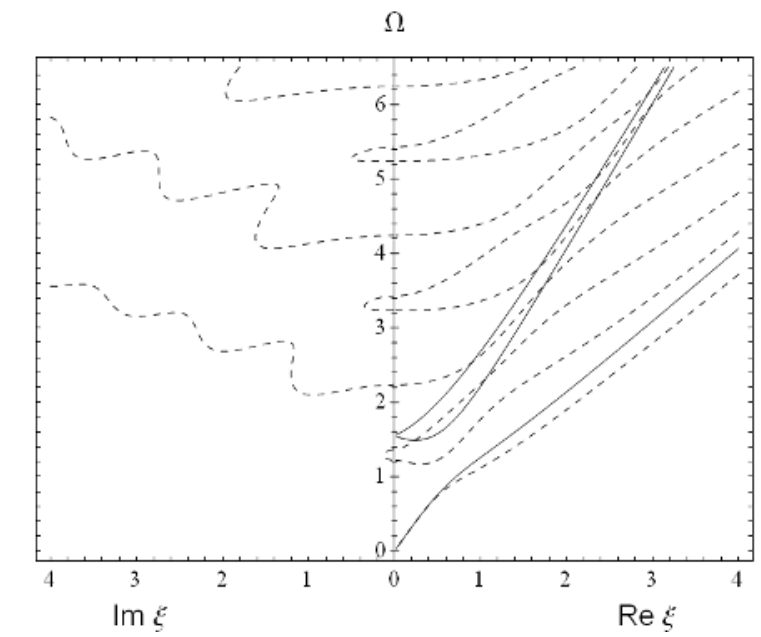

Fig. 4. Frequency spectrum of a three mode model (solid line), exact solution (dashed line).

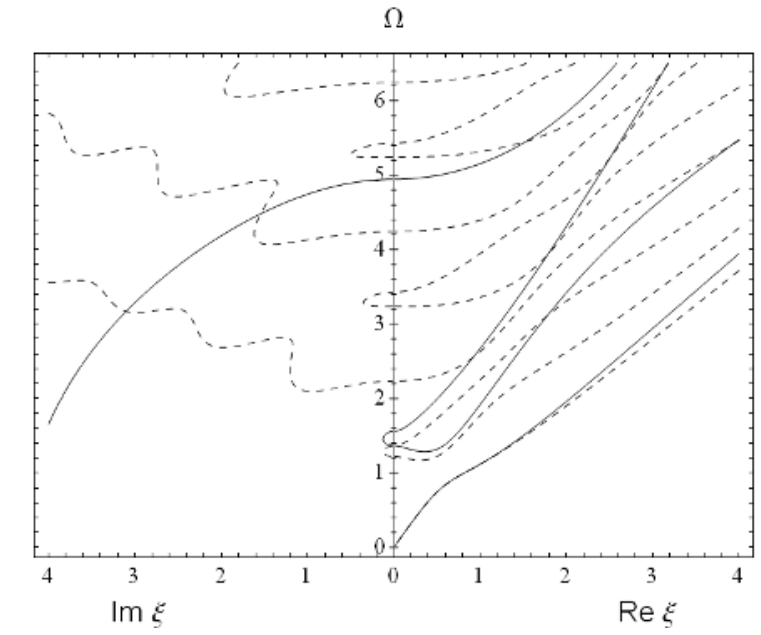

Fig. 5. Frequency spectrum of a three mode model (solid line), exact solution (dashed line).

Comparison of the frequency spectrum shown in figure 6 with figure 4 shows that the accuracy of the three branches in the Zachmanoglou-Volterra theory matches that of the first 
three branches of the four mode theory. That is, Zachmanoglou and Volterra simplified the four mode theory by reducing the number of partial differential equations and boundary conditions and the simplification did not have a negative impact on accuracy.

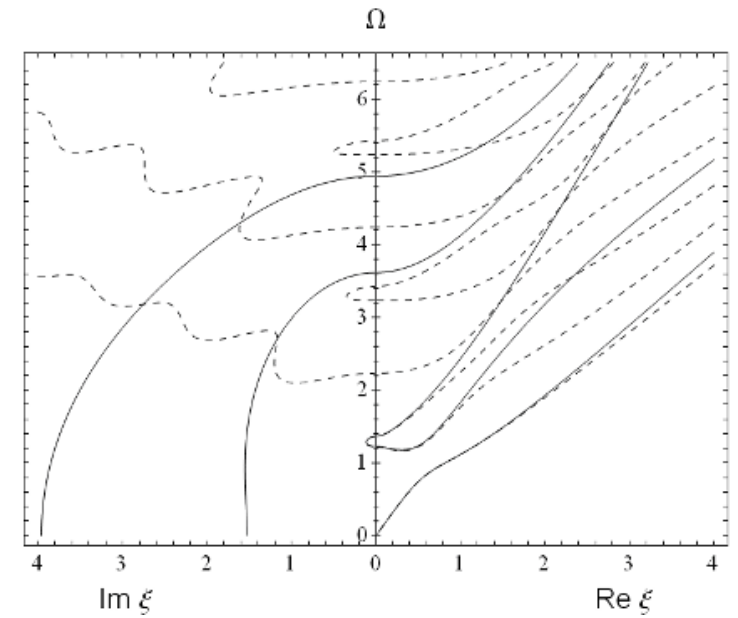

Fig. 6. Frequency spectrum of a five mode model (solid line), exact solution (dashed line).

The nature of the first branches of the multimode theories with increasing number of modes is of particular interest in the design of low frequency ultrasonic transducers and waveguides.

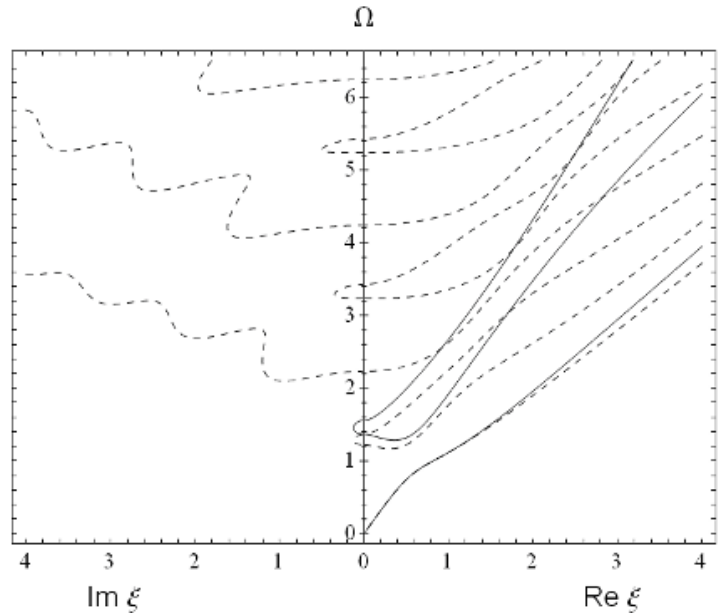

Fig. 7. Frequency spectrum of the Zachmanoglou-Volterra model (solid line), exact solution (dashed line).

Figure 8 shows the first branches of the spectral curves for the two mode, three mode and four mode models, together with the first branch of the exact Pochhammer-Chree solution. It is clear from this figure that the first branch of the approximate multimode theories 
approaches that of the exact Pochhammer-Chree solution with an increasing number of modes. The first branch of the five mode theory has not been included in figure 8 because it is too close to that of the four mode theory them to be easily distinguished from each other in the selected region for $\xi$.

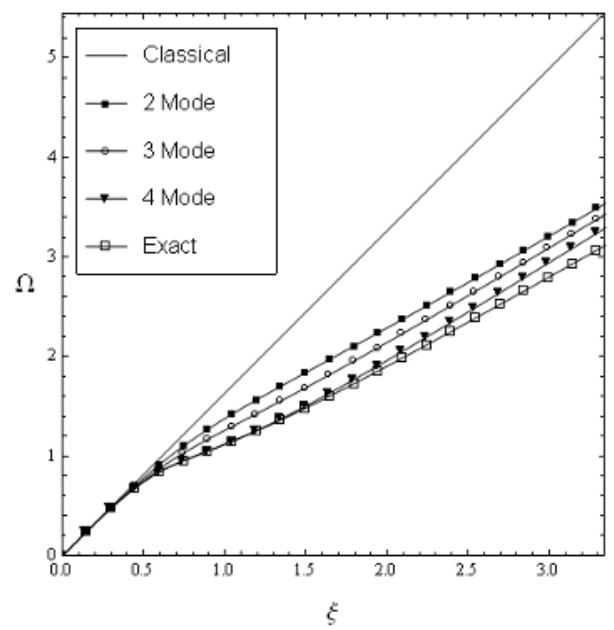

Fig. 8. First branches of the two, three, four mode models, and the exact solution.

\section{Conclusion}

In this article, a generalised theory for the derivation of approximate theories describing the longitudinal vibration of elastic bars has been proposed. The models outlined in this article represent a family of one dimensional hyperbolic differential equations, since all $u_{k}=u_{k}(x, t)$. An infinite number of these approximate theories have been introduced and the general procedure for derivation of the differential equations and boundary conditions for all the models considered has been exposed.

The approximate theories have been categorised as "unimode" or "multimode", and "plane cross sectional" or "non plane cross sectional" theories, based on the representation for longitudinal and lateral displacements (1)-(2). The classical, Rayleigh-Love and RayleighBishop models are all "unimode", "plane cross sectional" theories. Both the MindlinHermann and the three mode models are "multimode" theories. The Mindlin-Hermann model is a "plane cross sectional" theory, whereas the three-mode model is a "non plane cross sectional" theory. All models subsequent to the three-mode model (four-mode, fivemode, etc) are also "multimode", "non plane cross sectional" theories.

The orthogonality conditions have been found for the corresponding multimodal models which substantially simplify construction of the solution in terms of Green functions. The solution procedure for the Mindlin-Herrmann model has been presented, using the Fourier method. Using the method of two orthogonalities (presented here), it is possible to obtain the solution for all models considered in this article.

Finally, it has been shown that the accuracy of the approximate models discussed in this article approach that of the exact theory with increasing number of modes, based on a comparison of the frequency spectra with that of the exact Pochhammer-Chree frequency equation. 


\section{References}

Achenbach, J.D. (2005). Wave Propagation in Elastic Solids, Elsevier Science, ISBN 978-0-72040325-1, Amsterdam.

Bishop, R.E.D. (1952). Longitudinal Waves in Beams. Aeronautical Quarterly, Vol. 3, No. 2, 280-293.

Fedotov, I.A., Polyanin, A.D. \& Shatalov, M.Yu. (2007). Theory of Free and Forced Vibrations of a Rigid Rod Based on the Rayleigh Model. Doklady Physics, Vol. 52, No. 11, 607-612, ISSN 1028-3358.

Fedotov, I., Shatalov, M., Tenkam, H.M. \& Marais, J. (2009). Comparison of Classical and Modern Theories of Longitudinal Wave Propagation in Elastic Rods, Proceedings of the $16^{\text {th }}$ International Conference of Sound and Vibration, Krakow, Poland, 5 - 9 July, 2009.

Fedotov, I., Shatalov, M.A, Fedotova, T. \& Tenkam, H.M. (2010). Method of Multiple Orthogonalities for Vibration Problems, Current Themes in Engineering science 2009: Selected Presentations at the World Congress on Engineering 2009, AIP Conference Proceedings, Vol. 1220, 43-58, ISBN 978-0-7354-0766-4, American Institute of Physics.

Fung, Y.C. \& Tong, P. (2001). Classical and Computational Solid Mechanics, World Scientific, ISBN 978-981-02-3912-1, Singapore.

Gai, Y., Fedotov, I. \& Shatalov, M. (2007). Analysis of a Rayleigh-Bishop Model For a Thick Bar, Proceedings of the 2006 IEEE International Ultrasonics Symposium, 1915-1917, ISBN 1-4244-0201-8, Vancouver, Canada, 2 - 6 October, 2006, Institute of Electrical and Electronics Engineers.

Graff, K.F. (1991). Wave Motion in Elastic Solids, Dover Publications, ISBN 978-0-486-66745-6, New York.

Grigoljuk, E.I. \& Selezov, I.T. (1973). Mechanics of Solid Deformed Bodies, Vol. 5, Non-classical Theories of Rods, Plates and Shells, Nauka, Moscow. (In Russian).

Love, A.E.H. (2009). A Treatise on the Mathematical Theory of Elasticity, 2nd (1906) Edition, BiblioLife, ISBN 978-1-113-22366-1.

Mindlin, R.D. \& McNiven, H.D. (1960). Axially symmetric waves in elastics rods. Journal of Applied Mechanics, Vol. 27, 145-151, ISSN 00218936.

Porubov, A.V. (2003). Amplification of Nonlinear Strain Waves in Solids, World Scientific, ISBN 978-981-238-326-3, Singapore.

Rayleigh, J.W.S. (1945). Theory of sound, Vol I, Dover Publications, ISBN 978-0-486-60292-3, New York.

Zachmanoglou, E.C. \& Volterra, E. (1958). An Engineering Theory of Longitudinal Wave Propagation in Cylindrical Elastic Rods, Proceedings of the $3^{\text {rd }}$ US National Congress on Applied Mechanics, 239-245, Providence, Rhode Island, New York, 1958. 


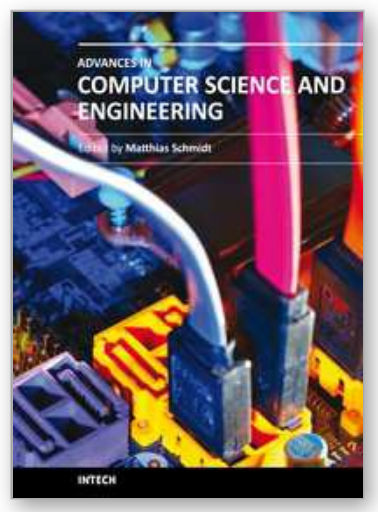

\author{
Advances in Computer Science and Engineering \\ Edited by Dr. Matthias Schmidt
}

ISBN 978-953-307-173-2

Hard cover, 462 pages

Publisher InTech

Published online 22, March, 2011

Published in print edition March, 2011

The book Advances in Computer Science and Engineering constitutes the revised selection of 23 chapters written by scientists and researchers from all over the world. The chapters cover topics in the scientific fields of Applied Computing Techniques, Innovations in Mechanical Engineering, Electrical Engineering and Applications and Advances in Applied Modeling.

\title{
How to reference
}

In order to correctly reference this scholarly work, feel free to copy and paste the following:

Michael Shatalov, Julian Marais, Igor Fedotov and Michel Djouosseu Tenkam (2011). Longitudinal Vibration of Isotropic Solid Rods: From Classical to Modern Theories, Advances in Computer Science and Engineering, Dr. Matthias Schmidt (Ed.), ISBN: 978-953-307-173-2, InTech, Available from:

http://www.intechopen.com/books/advances-in-computer-science-and-engineering/longitudinal-vibration-ofisotropic-solid-rods-from-classical-to-modern-theories

\section{INTECH}

open science | open minds

\section{InTech Europe}

University Campus STeP Ri

Slavka Krautzeka 83/A

51000 Rijeka, Croatia

Phone: +385 (51) 770447

Fax: +385 (51) 686166

www.intechopen.com

\section{InTech China}

Unit 405, Office Block, Hotel Equatorial Shanghai

No.65, Yan An Road (West), Shanghai, 200040, China 中国上海市延安西路65号上海国际贵都大饭店办公楼 405 单元

Phone: +86-21-62489820

Fax: $+86-21-62489821$ 
(C) 2011 The Author(s). Licensee IntechOpen. This chapter is distributed under the terms of the Creative Commons Attribution-NonCommercialShareAlike-3.0 License, which permits use, distribution and reproduction for non-commercial purposes, provided the original is properly cited and derivative works building on this content are distributed under the same license. 